\title{
PRIMORDIAL NUCLEOSYNTHESIS WITH VARYING FUNDAMENTAL CONSTANTS: A SEMIANALYTICAL APPROACH
}

\author{
Susana J. Landau, ${ }^{1,2,3}$ Mercedes E. Mosquera, ${ }^{1,4}$ and Hector Vucetich ${ }^{1}$ \\ Received 2004 November 9; accepted 2005 April 26
}

\begin{abstract}
Using the semianalytic method proposed by Esmailzadeh and coworkers we calculate the abundances of the light elements produced during primordial nucleosynthesis assuming that the gauge coupling constants of the fundamental interactions may vary. We analyze the dependence of the nucleon masses, nuclear binding energies, and cross sections involved in the calculation of the abundances with the fundamental constants assuming the chiral limit of QCD. We obtain the abundances of light elements as a function of the fundamental constants. Finally, using the observational data of $\mathrm{D},{ }^{3} \mathrm{He},{ }^{4} \mathrm{He}$, and ${ }^{7} \mathrm{Li}$, we estimate constraints on the variation of the fundamental constants between the time of primordial nucleosynthesis and the present. All observational abundances and the WMAP estimate of the baryon density can be fitted to the theoretical predictions with varying coupling constants. The possible systematic errors in the observational data preclude stronger conclusions.
\end{abstract}

Subject headings: cosmological parameters - early universe — nuclear reactions, nucleosynthesis, abundances

\section{INTRODUCTION}

Big bang nucleosynthesis (BBN) is one of the most important tools used to study the early universe. The model is simple and has only one free parameter, the density of baryonic matter, which can be determined by comparison between theoretical calculations and observations of the abundances of the light elements. On the other hand, data on cosmic microwave background (CMB) provide an alternative, independent method for determining $\Omega_{B} h^{2}$ (Spergel et al. 2003). Recently, the concordance between both methods has been investigated by many authors (Cyburt et al. 2003; Romano et al. 2003; Cuoco et al. 2004; Cyburt 2004; Coc et al. 2004a, 2004b). From the baryon density derived from the Wilkinson Microwave Anisotropy Probe (WMAP), the predicted abundances are highly consistent with the observed D but not with ${ }^{4} \mathrm{He}$ and ${ }^{7} \mathrm{Li}$; the predictions are more than observed. Such discrepancy is usually ascribed to unreported systematic errors in the observations of ${ }^{4} \mathrm{He}$ and ${ }^{7} \mathrm{Li}$. Indeed, more realistic determinations of the ${ }^{4} \mathrm{He}$ uncertainty implies a baryon density in line with the WMAP estimate (Cyburt 2004; Olive \& Skillman 2004). On the other hand, Richard et al. (2005) have pointed out that a better understanding of turbulent transport in the radiative zones of stars is needed for a better determination of the ${ }^{7} \mathrm{Li}$ abundance. However, if the systematic errors of ${ }^{4} \mathrm{He}$ and ${ }^{7} \mathrm{Li}$ are correctly estimated, we may have insight into new physics beyond the minimal BBN model, for example, new Neutron lifetime (Mathews et al. 2005), the super WIMP scenario (Feng et al. 2003), lepton asymmetry (Ichikawa et al. 2004), and varying constants (Bergström et al. 1999; Nollett \& Lopez 2002; Ichikawa \& Kawasaki 2002, 2004). Therefore, BBN not only is one of the most important tests of the big bang theory, but is also useful in obtaining stringent constraints on deviations from standard cosmology and on alternative theories to the standard model (SM) of fundamental interactions.

\footnotetext{
${ }^{1}$ Facultad de Ciencias Astronómicas y Geofísicas, Universidad Nacional de La Plata, Paseo del Bosque S/N 1900 La Plata, Argentina; mmosquera@carina .fcaglp.unlp.edu.ar, vucetich@fcaglp.unlp.edu.ar.

2 Departamento de Física, FCEyN, Universidad de Buenos Aires, Ciudad Universitaria - Pab. 1, 1428 Buenos Aires, Argentina; slandau@df.uba.ar.

${ }^{3}$ Member of CONICET.

${ }^{4}$ Fellow of CONICET.
}

Among these theories, there are some in which the gauge coupling constants may vary over cosmological timescales, like string-derived field theories (Wu \& Wang 1986; Maeda 1988; Barr \& Mohapatra 1988; Damour \& Polyakov 1994; Damour et al. 2002a, 2002b), related brane-world theories (Youm 2001a, 2001b; Palma et al. 2003; Brax et al. 2003), and (related or not) Kaluza-Klein theories (Kaluza 1921; Klein 1926; Weinberg 1983; Gleiser \& Taylor 1985; Overduin \& Wesson 1997). On the other hand, a theoretical framework in which only the finestructure constant varies was developed by Bekenstein (1982) and improved by Barrow et al. (2002). This model was generalized in order to study the time variation of the strong coupling constant (Chamoun et al. 2001). Different versions of the theories mentioned above predict different time behaviors of the gauge coupling constants. Thus, bounds obtained from astronomical and geophysical data are an important tool to test the validity of these theories.

The experimental research can be grouped into astronomical and local methods. The latter include geophysical methods such as the natural nuclear reactor that operated about $1.8 \times 10^{9}$ years ago in Oklo, Gabon (Damour \& Dyson 1996; Fujii et al. 2000, 2002 ), the analysis of natural long-lived $\beta$ decayers in geological minerals and meteorites (Dyson 1967; Sisterna \& Vucetich 1990; Smolliar 1996), and laboratory measurements such as comparisons of rates between clocks with different atomic number (Prestage et al. 1995; Sortais et al. 2000; Marion et al. 2003). The astronomical methods are based mainly on the analysis of spectra from high-redshift quasar absorption systems (Cowie \& Songaila 1995; Varshalovich et al. 1996; Webb et al. 1999, 2001; Murphy et al. 2001a, 2001b, 2003; Levshakov et al. 2002; Ivanchik et al. 2002, 2003; Bahcall et al. 2004). Although most of the previously mentioned experimental data gave null results, evidence of time variation of the fine-structure constant was reported recently in high-redshift quasar absorption systems (Webb et al. 1999, 2001; Murphy et al. 2001a, 2001b, 2003; Ivanchik et al. 2003). However, other recent independent analysis of similar data (Martínez Fiorenzano et al. 2003; Quast et al. 2004; Bahcall et al. 2004; Srianand et al. 2004) found no variation. On the other hand, measurements of molecular hydrogen (Ivanchik et al. 2002, 2003) reported a variation of the proton to electron mass $\mu=m_{p} / m_{e}$. 
The time variation of the gauge coupling constants in the early universe can be constrained using data from the cosmic microwave background (CMB; Battye et al. 2001; Avelino et al. 2000; Martins et al. 2002; Rocha et al. 2003) and the primordial abundances of light elements (Bergström et al. 1999; Nollett \& Lopez 2002; Ichikawa \& Kawasaki 2002, 2004).

The prediction of the light elements abundances $\left({ }^{4} \mathrm{He}, \mathrm{D},{ }^{7} \mathrm{Li}\right)$ produced during the first minutes of the universe can be calculated using numerical (Wagoner 1973; Kawano 1992) and analytical (Esmailzadeh et al. 1991; Mukhanov 2003) methods. Ichikawa \& Kawasaki (2002) modified the public code (Kawano 1992 ) in order to analyze the BBN scenario with varying gauge coupling constants. They considered a theoretical model taken from string theory in which the variation of the coupling constant is related to the expectation values of the dilaton field, and compared that to observational data. Consequently, the results they obtained are limited by the validity of this model. Furthermore, numerical calculations of the theoretical abundances of the light elements allowing only a variation of the fine-structure constant were performed by different authors (Bergström et al. 1999; Nollett \& Lopez 2002; Ichikawa \& Kawasaki 2004). Müller et al. (2004) performed an analytical study of ${ }^{4} \mathrm{He}$ abundance including variation of the gauge coupling constants. Moreover, the change in the abundance of ${ }^{4} \mathrm{He}$ due to variable mass in fivedimensional theories was analyzed by Anchordoqui et al. (1996). Finally, the effect of considering nonextensive thermostatistics has been analyzed by various authors ( Torres et al. 1997; Pessah et al. 2001; Pessah \& Torres 2001).

In this work, we follow the semianalytical method proposed by Esmailzadeh et al. (1991) to study the effect of a possible variation of the values of the three gauge coupling constants of the standard model of particle interactions between primordial nucleosynthesis and the present. Even though the semianalytical method gives results 1 order of magnitude less accurate than the calculations performed with the numerical code, it is very useful to find out the dependence of the abundances and temperatures on the fundamental constants, which is one of the principal aims of this work.

We do not assume any of the theoretical models for varying constants mentioned above. Motivated by theoretical predictions and observational data, we study the formation of the light elements in the early universe assuming that the values of the gauge coupling constants of the fundamental interactions (electromagnetic, strong, and weak) may be different from their actual value. Thus, our approach is a phenomenological one and our results will be model-independent. Furthermore, we assume the chiral limit of QCD to analyze the dependence of nucleon masses, binding energies, and cross sections on the strong interaction coupling constants. The gauge coupling constants of $U(1)$, $S U(2)$, and $S U(3)$ (namely, $\alpha_{1}, \alpha_{2}$, and $\alpha_{3}$ ) are related to the fine-structure constant $\alpha$, the QCD energy scale $\Lambda_{\mathrm{QCD}}$, and the Fermi coupling constant $G_{\mathrm{F}}$ through the following equations:

$$
\begin{gathered}
\alpha^{-1}(E)=\frac{5}{2} \alpha_{1}^{-1}(E)+\alpha_{2}^{-1}(E), \\
\Lambda_{\mathrm{QCD}}=E \exp \left[-\frac{2 \pi}{7} \alpha_{3}^{-1}(E)\right], \\
G_{\mathrm{F}}=\frac{\pi \alpha_{2}\left(M_{Z}\right)}{\sqrt{2} M_{Z}^{2}},
\end{gathered}
$$

where $E$ refers to the energy scale and $M_{Z}$ refers to the boson $Z$ mass. Actually, we study the dependence of the different phys- ical quantities involved in the calculation of the primordial abundances with $\alpha, \Lambda_{\mathrm{QCD}}$, and $G_{\mathrm{F}}$.

Almost all of the observational and experimental data are consistent with no variation of the constants (Landau \& Vucetich 2002). Moreover, the reported variations (Murphy et al. 2003; Ivanchik et al. 2003) are very small $\left(\Delta \alpha_{i} / \alpha_{i} \sim 10^{-5}\right)$. Therefore, in order to find out the dependences of relevant physical quantities with $\alpha, \Lambda_{\mathrm{QCD}}$, and $G_{\mathrm{F}}$, we perform a Taylor expansion to first order in each case as

$$
\begin{aligned}
& \Delta Q=\left.\frac{\partial Q}{\partial \alpha}\right|_{\left(\alpha^{\mathrm{tod}}, \Lambda_{\mathrm{QCD}}^{\mathrm{tod}}, G_{\mathrm{F}}^{\mathrm{tod}}\right)} \Delta \alpha \\
& +\left.\frac{\partial Q}{\partial \Lambda_{\mathrm{QCD}}}\right|_{\left(\alpha^{\mathrm{tod}}, \Lambda_{\mathrm{QCD}}^{\mathrm{tod}}, G_{\mathrm{F}}^{\mathrm{tod}}\right)} \Delta \Lambda_{\mathrm{QCD}}+\left.\frac{\partial Q}{\partial G_{\mathrm{F}}}\right|_{\left(\alpha^{\mathrm{tod},}, \Lambda_{\mathrm{QCD}}^{\mathrm{tod}}, G_{\mathrm{F}}^{\mathrm{tod}}\right)} \Delta G_{\mathrm{F}},
\end{aligned}
$$

where $Q$ refers to the physical quantities involved in the nucleosynthesis calculation such as nucleon and nucleus masses, nuclear binding energies, cross sections, and abundances of the elements. (Here and elsewhere, the abbreviation "tod" in a superscript refers to conditions today.)

In the standard picture, the only free parameter of the nucleosynthesis calculation is the density of baryonic matter, $\Omega_{B} h^{2}$. This quantity has been determined with great accuracy using data from the CMB provided by WMAP (Spergel et al. 2003). On the other hand, the baryon density can also be estimated using data provided by galaxy surveys (SDSS, $2 \mathrm{dF}$ ) and X-ray satellites (Chandra X-Ray Observatory, XMM-Newton, Röntgensatellit [ROSAT], and $A S C A$ ). In Appendix A we combine different data to obtain an estimate of $\Omega_{B} h^{2}$ independent of the WMAP estimate. Therefore, we approach the problem by studying the dependences of all physical quantities and abundances on both the fundamental constants and the baryon density. Thus, we will obtain the uncertainties of the abundances of the light elements as function of the variations of the fundamental constants with respect to their actual value and as function of the variation of $\Omega_{B} h^{2}$ with respect to the WMAP estimate (Spergel et al. 2003). On the other hand, we also compare the predicted theoretical expressions for the abundances to observational data and include independent estimates of the baryon density in the analysis (see $\S 5)$.

Furthermore, in $\S 2$, we calculate the dependence of the nucleon masses and binding energies on the fundamental constants, and in $\S 3$, the corresponding dependence of the relevant scattering cross sections. We have carried out these calculations in some detail, since there are several subtle points in these dependences that will be clearly exhibited in the final results. In $\S 4$ we apply the semianalytical method proposed by Esmailzadeh et al. (1991) to calculate the abundances of the light elements and their dependence on the fundamental constants. In $\S 5$ we briefly describe the observational data and the results of comparison with the theoretical predictions calculated in this work. We also discuss our conclusions.

\section{MASSES AND BINDING ENERGIES OF LIGHT ELEMENTS}

In this section we analyze the dependence of the nucleon masses, nuclear binding energies, and nuclei masses of the light elements with the fundamental constants $\alpha$ and $\Lambda_{\mathrm{QCD}}$. The weak interaction contribution is too small to produce any observable consequences (Haugan \& Will 1976; Chamoun \& Vucetich 2002). 
The dependence of the hadronic masses and nuclear binding energies with the QCD coupling constant $\alpha_{3}$ or the QCD scale parameter $\Lambda_{\mathrm{QCD}}$ depends on the model of hadronic interactions considered. However, if we assume that the quark masses are null, an assumption called in the literature a chiral limit, there is a only a single parameter in the theory, namely the QCD scale parameter $\Lambda_{\mathrm{QCD}}$. Even though great efforts (Beane \& Savage 2003a, 2003b; Epelbaum et al. 2003; Flambaum \& Shuryak 2002, 2003; Dmitriev \& Flambaum 2003; Dmitriev et al. 2004; Olive et al. 2002) have been made to analyze the dependence of nucleon masses and binding energies on $\Lambda_{\mathrm{QCD}}$ beyond the chiral limit, this task is not trivial and is highly model-dependent.

On the other hand, from simple dimensional analysis (Stevenson 1981), it follows that in a theory with only one relevant parameter all static observables with a dimension of mass must be proportional to this parameter, which in our case is $\Lambda_{\mathrm{QCD}}$. More precisely, any quantity $\sigma$ with units of $E^{D}$ (where $E$ means energy) must satisfy an equation of the form

$$
\sigma=\Lambda_{\mathrm{QCD}}^{D} f\left[\frac{Q}{\Lambda_{\mathrm{QCD}}}\right]
$$

where $Q$ is a quantity specifying the energy scale. Furthermore, for static quantities such as nucleon masses the previous equation takes the form

$$
\sigma=\Lambda_{\mathrm{QCD}}^{D} f\left[\frac{\sigma}{\Lambda_{\mathrm{QCD}}}\right]
$$

since the only scale parameter is $\sigma$ itself. The solution of equation (6) reads

$$
\sigma=\Lambda_{\mathrm{QCD}}^{D} X
$$

where $X$ is a dimensionless numerical constant. In this way, all low-energy static quantities will satisfy an equation of the form of equation (7). Moreover, all nucleon masses and energies will have a linear dependence

$$
m_{N} \sim \epsilon_{B} \sim \Lambda_{\mathrm{QCD}}
$$

and all nuclear radii will satisfy

$$
R \sim \Lambda_{\mathrm{QCD}}^{-1}
$$

since we use units where $\hbar=c=1$ for this analysis. The chiral limit was previously considered by Sisterna \& Vucetich (1990) studying time variations of fundamental constants in planetary phenomena.

The mass of the nucleons can be written as a sum of two contributions, the electromagnetic contribution $m_{N}^{C}$ and the strong interaction contribution $m_{N}^{S}$,

$$
m_{N}=m_{N}^{C}+m_{N}^{S}
$$

\begin{tabular}{|c|c|}
\hline Nucleon/Nucleus & $P\left(\times 10^{-4}\right)$ \\
\hline$n_{p} \ldots$ & 6.71 \\
\hline ........................ & -1.38 \\
\hline . & 2.67 \\
\hline Т & 1.32 \\
\hline - & 1.05 \\
\hline 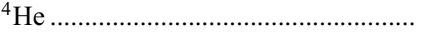 & 0.66 \\
\hline${ }^{6} \mathrm{Li}$ & 1.50 \\
\hline${ }^{7} \mathrm{Li}$ & 1.14 \\
\hline${ }^{7} \mathrm{Be}$ & 2.30 \\
\hline
\end{tabular}

The electromagnetic contribution depends on the nuclear radius $R$ as

$$
\epsilon_{C}=\frac{Z}{4 \pi \epsilon_{0}} \frac{e^{2}}{R}
$$

TABLE 1

Therefore, the electromagnetic contribution to the nucleon mass in the chiral limit has the following dependence with $\Lambda_{\mathrm{QCD}}$ :

$$
m_{N}^{C} \sim \Lambda_{\mathrm{QCD}}
$$

Cottingham (1963) used perturbation theory to calculate the electromagnetic self-energy of a nucleon $m_{N}$ to first order in $\alpha$

$$
m_{N}^{C} \sim K \alpha,
$$

where $K$ can be expressed as a function of Sachs form factors $G_{E, M}^{N}$, which can be calculated from measurements of electronnucleon scattering. However, the strong interaction contribution to the mass in the chiral limit is proportional to $\Lambda_{\mathrm{QCD}}$. Therefore, we can write

$$
\begin{aligned}
& m_{N}^{C}=m_{N}^{C} \frac{\alpha}{\alpha^{\text {tod }}} \frac{\Lambda_{\mathrm{QCD}}}{\Lambda_{\mathrm{QCD}}^{\text {tod }}}, \\
& m_{N}^{S}=m_{N}^{S} \frac{\Lambda_{\mathrm{QCD}}}{\Lambda_{\mathrm{QCD}}^{\text {tod }}} .
\end{aligned}
$$

After performing a Taylor expansion to first order, as explained in $\S 1$, and using equations (14) and (15), we obtain the dependence of the nucleon masses with the fundamental constants

$$
\frac{\delta m_{N}}{m_{N}}=\frac{m_{N}^{C}}{m_{N}} \frac{\delta \alpha}{\alpha}+\frac{\delta \Lambda_{\mathrm{QCD}}}{\Lambda_{\mathrm{QCD}}}=P \frac{\delta \alpha}{\alpha}+\frac{\delta \Lambda_{\mathrm{QCD}}}{\Lambda_{\mathrm{QCD}}} .
$$

The values of $P$ are shown in Table 1 .

Next, we analyze the dependence of the nuclei masses on $\alpha$ and $\Lambda_{\mathrm{QCD}}$. As we did for nucleons, we perform a Taylor expansion to first order to obtain for a nucleus of mass $m_{x}$ the expression

$$
\frac{\delta m_{x}}{m_{x}}=(A-Z) \frac{m_{n}}{m_{x}} \frac{\delta m_{n}}{m_{n}}+Z \frac{m_{p}}{m_{x}} \frac{\delta m_{p}}{m_{p}}-\frac{\epsilon_{x}}{m_{x}} \frac{\delta \epsilon_{x}}{\epsilon_{x}} .
$$

In the more general case, the binding energy $\left(\epsilon_{x}\right)$ can be written as a sum of two terms: the electromagnetic contribution $\left(\epsilon_{C}\right)$ and the strong interaction contribution $\left(\epsilon_{S}\right)$, as $\epsilon_{x}=\epsilon_{C}+\epsilon_{S}$. In the cases of nuclei with only one proton ( $D$ and $T$ ), there is no electromagnetic interaction and therefore the electromagnetic contribution $\left(\epsilon_{C}\right)$ is null. The same arguments that were used to 
obtain equations (14) and (15) can be applied to the binding energy to obtain

$$
\frac{\delta \epsilon_{x}}{\epsilon_{x}}=\frac{\epsilon_{C}}{\epsilon_{x}} \frac{\delta \alpha}{\alpha}+\frac{\delta \Lambda_{\mathrm{QCD}}}{\Lambda_{\mathrm{QCD}}} .
$$

Inserting this last expression in equation (17), we obtain the general expression for the dependence of a nucleus mass with $\alpha$ and $\Lambda_{\mathrm{QCD}}$

$$
\frac{\delta m_{x}}{m_{x}}=P \frac{\delta \alpha}{\alpha}+\frac{\delta \Lambda_{\mathrm{QCD}}}{\Lambda_{\mathrm{QCD}}} .
$$

The values $P$ for different nuclei are shown in Table 1 .

\section{THERMONUCLEAR REACTION RATES}

In this section we calculate the thermonuclear reaction rates as functions of fundamental constants. We also show the dependence of the reaction rates with the baryon density $\rho_{B}=\Omega_{B} h^{2}$. Following Esmailzadeh et al. (1991) we can write the thermonuclear reaction rate as:

$[i j \rightarrow k l]=\rho_{B} N_{\mathrm{A}}\langle\sigma v\rangle=0.93 \times 10^{-3} \Omega_{B} h^{2} T_{9}^{3} N_{\mathrm{A}}\langle\sigma v\rangle \mathrm{s}^{-1}$,

where $\sigma$ is the cross section, $v$ is the relative velocity, $\rho_{B}=$ $0.93 \times 10^{-3} \Omega_{B} h^{2} T_{9}^{3} \mathrm{~g} \mathrm{~cm}^{-3}$ is the density of baryonic matter, $N_{\mathrm{A}}$ is Avogadro's number per gram, and $T_{9}$ is the temperature in units of $10^{9} \mathrm{~K}$.

Using a Maxwell-Boltzmann distribution in velocities, the Boltzmann averaged cross section, $\langle\sigma v\rangle$ can be expressed as

$$
\langle\sigma v\rangle=\left(\frac{\mu}{2 \pi k T}\right)^{3 / 2} \int e^{-\mu v^{2} / 2 k T} v \sigma(E) d^{3} v .
$$

We need to find $\delta[i j \rightarrow k l] /[i j \rightarrow k l]$ as a function of the relative variations of the fundamental constants $\left(\delta \alpha / \alpha, \delta \Lambda_{\mathrm{OCD}} /\right.$ $\Lambda_{\mathrm{QCD}}, \delta G_{\mathrm{F}} / G_{\mathrm{F}}$ ) and $\delta \Omega_{B} h^{2} / \Omega_{B} h^{2}$, the relative variation of the value of the baryon density with respect to WMAP estimate $\Omega_{B} h^{2}=0.0224$ (Spergel et al. 2003). The temperature does not depend on the values of the fundamental constants, but the final temperature of each stage does, and therefore we can write

$$
\frac{\delta[i j \rightarrow k l]}{[i j \rightarrow k l]}=\frac{\delta \Omega_{B} h^{2}}{\Omega_{B} h^{2}}+3 \frac{\delta T_{9}^{f}}{T_{9}^{f}}+\frac{\delta\langle\sigma v\rangle}{\langle\sigma v\rangle},
$$

where $T_{9}^{f}=f\left(\alpha, \Lambda_{\mathrm{QCD}}, G_{\mathrm{F}}\right)$ for all the reaction rates. On the other hand, $\delta\langle\sigma v\rangle /\langle\sigma v\rangle$ depends on the fundamental constants through the masses of the nucleons and light nuclei and the form factor of the reactions. In the general case, there are no analytic expressions for $\sigma(E)$ derived from "first principles." We suggest several expressions that attempt to fit $\sigma(E)$, according to the elements in the reactions .

\subsection{Cross Sections for Charged Particle Reactions}

The cross section for charged particle reactions is given by Fowler et al. $(1967,1975)$ and Wagoner et al. (1967),

$$
\sigma=\frac{S(E)}{E} e^{-2 \pi \alpha Z_{1} Z_{2} \sqrt{\mu c^{2} / 2 E}},
$$

where $Z_{i}$ is the charge of the $i$ th particle, $\mu=m_{1} m_{2} /\left(m_{1}+m_{2}\right)$ is the reduced mass, $E$ is the energy, and $S(E)$ is the form factor.
TABLE 2

Dependence of Radiative Captures on $\alpha\left(f(\alpha)=\sum b_{i}\left[\alpha / \alpha^{\text {tod }}-1\right]\right)$

\begin{tabular}{ccccrrr}
\hline \hline Reaction & $b_{0}$ & $b_{1}$ & $b_{2}$ & \multicolumn{1}{c}{$b_{3}$} & \multicolumn{1}{c}{$b_{4}$} & \multicolumn{1}{c}{$b_{5}$} \\
\hline${ }^{3} \mathrm{H}(\alpha, \gamma){ }^{7} \mathrm{Li} \ldots \ldots \ldots \ldots . . . .$. & 1 & 1.372 & 0.502 & 0.183 & 0.269 & -0.218 \\
${ }^{3} \mathrm{He}(\alpha, \gamma){ }^{7} \mathrm{Be} \ldots \ldots \ldots \ldots$. & 1 & 2.148 & 0.669 & -5.566 & -10.630 & -5.730 \\
\hline
\end{tabular}

The dependence of the cross sections for charged-particle reactions have been previously analyzed (Bergström et al. 1999; Nollett\& Lopez 2002). In particular, Nollett\& Lopez (2002) improved the analysis and studied the form factor as a function of $\alpha$. In this paper, we use the criteria established by these authors to analyze the dependence of the form factor with $\alpha$.

Next, we analyze the dependence of the form factor on $\Lambda_{\mathrm{QCD}}$ using dimensional arguments and the chiral limit. The units of the cross section are $\mathrm{cm}^{2}$, and therefore it follows that in a theory with massless quarks $\sigma \sim \Lambda_{\mathrm{OCD}}^{-2}$. The only quantity of equation (23) that has units is the factor $S(E) / E$ and thus we obtain

$$
S(E) \sim \Lambda_{\mathrm{QCD}}^{-1}
$$

This is valid for all charged-particle reactions. The exact dependence of the form factor $S(E)$ on the energy is unknown. However, as it is usually done in the literature (Fowler et al. 1967, 1975; Wagoner et al. 1967), we can do a Maclaurin expansion,

$$
S(E)=S(0)\left[1+\left(\frac{d S}{d E}\right)_{E=0} \frac{1}{S(0)} E+\frac{1}{2}\left(\frac{d^{2} S}{d E^{2}}\right)_{E=0} \frac{1}{S(0)} E^{2}\right],
$$

where $d S / d E$ and $d^{2} S / d E^{2}$ are expressed in barn and barn $\mathrm{MeV}^{-1}$, respectively. The terms inside the parentheses have no dimensions, therefore

$$
S(0) \sim \Lambda_{\mathrm{QCD}}^{-1}
$$

The dependence of the charged-particle cross sections on $\alpha$ has been analyzed by Nollett\& Lopez (2002), yielding

$$
S(0) \sim \alpha .
$$

\begin{tabular}{|c|c|}
\hline Reaction & Multiplied by: \\
\hline Charged particle reaction rates... & $\alpha / \alpha^{\text {tod }}$ \\
\hline Photon emission..... & $\alpha / \alpha^{\text {tod }}$ \\
\hline${ }^{2} \mathrm{H}(d, p)$ & $1+0.16-0.16\left(\alpha / \alpha^{\mathrm{tod}}\right)$ \\
\hline${ }^{3} \mathrm{He}(n, p)$ & $1-0.30+0.30\left(\alpha / \alpha^{\text {tod }}\right)$ \\
\hline${ }^{3} \mathrm{He}(d, p)$ & $1+0.09-0.09\left(\alpha / \alpha^{\text {tod }}\right)$ \\
\hline${ }^{7} \operatorname{Li}(p, \alpha)$ & $1+0.18-0.18\left(\alpha / \alpha^{\text {tod }}\right)$ \\
\hline${ }^{7} \mathrm{Be}(n, p)$ & $1-0.20+0.20\left(\alpha / \alpha^{\mathrm{tod}}\right)$ \\
\hline
\end{tabular}

Furthermore, it follows that all radiative capture rates should be multiplied by a factor $\alpha / \alpha^{\text {tod }}$, except the reactions $\mathrm{T}(\alpha \gamma)^{7} \mathrm{Li}$ and ${ }^{3} \mathrm{He}(\alpha \gamma)^{7} \mathrm{Be}$. These cross sections should be multiplied by $f(\alpha)=\sum b_{i}\left[\alpha / \alpha^{\text {tod }}-1\right]$ (see Table 2). Finally, in the cases in which the reaction produces two charged particles, the cross section should by multiplied by $1-b+b\left(\alpha / \alpha^{\text {tod }}\right)$ (see Table 3$)$.

TABLE 3

Dependence of Different Kinds of Reactions Rates on $\alpha$ 
TABLE 4

Charged Particle Reaction Rates I

\begin{tabular}{|c|c|}
\hline Reaction & $\begin{array}{l}\text { Reaction Rate } \\
\left(\mathrm{s}^{-1}\right)\end{array}$ \\
\hline${ }^{3} \mathrm{H}(p, \gamma){ }^{4} \mathrm{He}$. & $\begin{array}{l}1.14 \times 10^{-7} \Theta[\Sigma(0)]^{2} e^{-9.55 \times 10^{10}\left(\Psi / T_{9}\right)^{1 / 3}} \\
\quad \times P_{I B}\left(\Psi, T_{9}, 4.36,4.14,1.26,3.35,2.61\right)\end{array}$ \\
\hline${ }^{2} \mathrm{H}(d, n){ }^{3} \mathrm{He} .$. & $\begin{array}{l}2.26 \times 10^{-3} \Theta \Sigma(0) e^{-9.55 \times 10^{10}\left(\Psi / T_{9}\right)^{1 / 3}} \\
\quad \times P_{I B}\left(\Psi, T_{9}, 4.36,1.96,0.6,-0.206,-0.16\right)\end{array}$ \\
\hline${ }^{2} \mathrm{H}\left({ }^{3} \mathrm{He}, p\right){ }^{4} \mathrm{He} \ldots \ldots \ldots \ldots$ & $\begin{array}{l}0.39 \Theta \Sigma(0.09) e^{-1.52 \times 10^{11}\left(\Psi / T_{9}\right)^{1 / 3}-\left(507.36 T_{9} \alpha\right)^{2}} \\
\quad \times P_{I B}\left(\Psi, T_{9}, 2.75,-2.16,-0.42,13.5,6.58\right) \\
\quad+2.63 \times 10^{-8} \Xi(1 / 2) \Sigma(0.09) T_{9}^{5 / 2} e^{-1.76 T_{9}^{-1}}\end{array}$ \\
\hline${ }^{3} \mathrm{H}(d, n)^{4} \mathrm{He} .$. & $\begin{array}{l}0.49 \Theta \Sigma(0) e^{-9.55 \times 10^{10}\left(\Psi / T_{9}\right)^{1 / 3}-\left(1141.67 T_{9} \alpha\right)^{2}} \\
\quad \times P_{I B}\left(\Psi, T_{9}, 4.36,3.78,1.16,46.8,3.64 \times 10^{11}\right) \\
\quad+3.39 \times 10^{-8} \Xi(1 / 2) \Sigma(0) T_{9}^{7 / 3} e^{-0.523 T_{9}^{-1}}\end{array}$ \\
\hline${ }^{2} \mathrm{H}(d, n)^{3} \mathrm{H} \ldots \ldots \ldots \ldots$ & $\begin{array}{l}2.37 \times 10^{-3} \Theta \Sigma(0.16) e^{-9.55 \times 10^{10}\left(\Psi / T_{9}\right)^{1 / 3}} \\
\quad \times P_{I B}\left(\Psi, T_{9}, 4.36,1.16,0.35,-0.051,-0.04\right)\end{array}$ \\
\hline${ }^{2} \mathrm{H}(\alpha, \gamma){ }^{6} \mathrm{Li} .$. & $\begin{array}{l}1.88 \times 10^{-10} \Theta[\Sigma(0)]^{2} e^{-1.52 \times 10^{11}\left(\Psi / T_{9}\right)^{1 / 3}} \\
\quad \times P_{I B}\left(\Psi, T_{9}, 2.75,-9.9,8.85,-2.43,-1.19\right) \\
\quad+8.27 \times 10^{-39} \Xi(3 / 2)[\Sigma(0)]^{2} T_{9}^{3 / 2} e^{-8.228 T_{9}^{-1}}\end{array}$ \\
\hline $\mathrm{H}\left({ }^{6} \mathrm{Li}, \alpha\right)^{3} \mathrm{H} \ldots \ldots \ldots \ldots$ & $\begin{array}{l}0.20 \Theta \Sigma(0) e^{-1.99 \times 10^{11}\left(\Psi / T_{9}\right)^{1 / 3}-\left(24.94 T_{9} \alpha\right)^{2}} \\
\quad P_{I B}\left(\Psi, T_{9}, 2.10,-0.14,-0.02,0.033,0.012\right) \\
\quad+4.53 \times 10^{-8} \Xi(1 / 2) \Sigma(0) T_{9}^{2} e^{-21.82 T_{9}^{-1}} \\
\quad+6.68 \times 10^{-34} \Xi(3 / 2) \Sigma(0) T_{9}^{3 / 2} e^{-17.76 T_{9}^{-1}}\end{array}$ \\
\hline
\end{tabular}

Note. $-\Theta=\Omega_{B} h^{2} T_{9}^{7 / 3} \alpha^{1 / 3} \mu^{-1 / 3}, \Psi=\mu \alpha^{2}, \Xi(b)=\Omega_{B} h^{2} \mu^{-b}$,

$P_{I B}\left(\Psi, T_{9}, c_{1}, c_{2}, c_{3}, c_{4}, c_{5}\right)=1+c_{1} \times 10^{-12} \Psi^{-1 / 3} T^{1 / 3}{ }_{9}+c_{2} \times 10^{10} \Psi^{1 / 3} T_{9}^{2 / 3}+$ $c_{3} T_{9}+c_{4} \times 10^{20} \Psi^{2 / 3} T_{9}^{4 / 3}+c_{5} \times 10^{10} \Psi^{1 / 3} T_{9}^{5 / 3}$.

We insert the expression for $\sigma(E)$ into the equation (21) in order to calculate the Boltzmann averaged cross sections,

$$
\begin{aligned}
\langle\sigma v\rangle= & \sqrt{\frac{8}{\mu \pi}}(k T)^{-1 / 2} \sum_{i=0}^{2} \frac{(k T)^{i}}{i !}\left(\frac{d^{i} S}{d E^{i}}\right)_{E=0} \\
& \times \int_{0}^{\infty} y^{i} e^{-y-\xi y^{-1 / 2}} d y,
\end{aligned}
$$

where $\xi=2 \pi \alpha Z_{1} Z_{2}\left(\mu c^{2} / 2 k T\right)^{1 / 2}$ and the masses are in kilograms. The integrals are calculated in Bergström et al. (1999). Tables 4 and 5 show the dependence of charged-particles reaction rates on the fundamental constants.

\subsubsection{Cutoff Factor}

The truncated Maclaurin series we have used for $S(E)$ diverges at high energy. Thus, it is important to include a cutoff factor for nonresonant reaction rates so that they can be used at any energy. The next term in the expansion for $S(E)$ would be proportional to $E^{3} \sim T^{2}$, so as is proposed in the literature (Fowler et al. $1967,1975)$ we consider a cutoff factor $f_{\mathrm{co}}=e^{-\left(T_{9} / T_{\mathrm{co}}\right)^{2}}$, where $T_{\text {co }} \sim E_{r} / \alpha$ and $E_{r}$ is the resonant energy (Fowler et al. 1967, 1975). Therefore, we multiply the expression (28) by a factor

$$
f_{\mathrm{co}}=e^{-\left(\alpha T_{9} / E_{r}\right)^{2}} .
$$

This correction is relevant for the reactions ${ }^{3} \mathrm{He}(d, p){ }^{4} \mathrm{He}$, ${ }^{3} \mathrm{H}(d, n){ }^{4} \mathrm{He},{ }^{6} \mathrm{Li}(p, \alpha){ }^{3} \mathrm{H},{ }^{6} \mathrm{Li}(\alpha, p){ }^{10} \mathrm{Be}$, and ${ }^{7} \mathrm{Li}(p, \alpha){ }^{4} \mathrm{He}$.

\subsubsection{Alternative Expression for the Form Factor}

In the $\mathrm{MeV}$ range the cross section form factor varies considerably. In this range the truncated Maclaurin series is not satisfactory, so it is convenient (Fowler et al. 1967, 1975) to use for $S(E)$ an expression of the form $S(E)=S(0) e^{-a E}$. In such way, the cross sections are given by

$$
\sigma(E)=\frac{S(0)}{E} e^{-a E} e^{-2 \pi \alpha Z_{1} Z_{2} \sqrt{\mu c^{2} / 2 E}}
$$

where $a$ has no dependence on the fundamental constants. The quantities with units in equation 30 are $S(0)$ and $E$; therefore, in the chiral limit we have $S(0) \sim \Lambda_{\mathrm{QCD}}^{-1}$. In this way, the Boltzmann cross section (eq. [21]) yields

$$
\begin{aligned}
\langle\sigma v\rangle= & \frac{8}{\sqrt{6}}\left(\frac{\mu}{k T}\right)^{3 / 2} \frac{S(0) k T}{a k T+1}\left(\frac{\xi_{a}^{2}}{4}\right)^{1 / 6} e^{-3\left(\xi_{a}^{2} / 4\right)^{1 / 3}} \\
& \times\left[1+\frac{5}{36}\left(\frac{\xi_{a}^{2}}{4}\right)^{-1 / 3}\right] \mathrm{cm}^{3} \mathrm{~s}^{-1},
\end{aligned}
$$

where $\xi_{a}=\xi(a k T+1)^{1 / 2}=2 \pi \alpha Z_{1} Z_{2}\left[\left(\mu c^{2} / 2 k T\right)(a k T+1)\right]^{1 / 2}$. This alternative expression for nonresonant reaction rates is relevant for the reactions: ${ }^{6} \mathrm{Li}(p, \gamma){ }^{7} \mathrm{Be},{ }^{3} \mathrm{H}(\alpha, \gamma){ }^{7} \mathrm{Li}$, and ${ }^{3} \mathrm{He}(\alpha, \gamma){ }^{7} \mathrm{Be}$.

\subsection{Resonant Charged-Particle Reaction Rates}

\begin{tabular}{|c|c|}
\hline Reaction & $\begin{array}{l}\text { Reaction Rate } \\
\left(\mathrm{s}^{-1}\right)\end{array}$ \\
\hline${ }^{3} \mathrm{H}(\alpha, \gamma){ }^{7} \mathrm{Li} \ldots \ldots \ldots \ldots$ & $\begin{array}{l}\Theta[\Sigma(0)]^{2} P_{L N}\left(\left[\alpha-\alpha^{\mathrm{tod}}\right] / \alpha^{\mathrm{tod}}-\alpha^{\mathrm{today}}, 3.17,0.50,0.18,0.27,-0.22\right) \\
\quad \times\left\{7.47 \times 10^{-6} e^{-1.52 \times 10^{11}\left(\Psi / T_{9}\right)^{1 / 3}} P_{I B}\left(\Psi, T_{9}, 2.75,-0.76,-0.15,0.36,0.18\right)+2.68 \times 10^{-5} T_{9_{v}}^{-5 / 6} e^{-6.69 \times 10^{10}\left(\Psi T_{9_{v}} / T_{9}\right)^{1 / 3}}\right\}, v=1.59\end{array}$ \\
\hline $\mathrm{H}\left({ }^{7} \mathrm{Li}, \alpha\right){ }^{4} \mathrm{He} \ldots \ldots \ldots$ & $\begin{array}{l}3.33 \times 10^{-3} \Theta \Sigma(0) e^{-1.99 \times 10^{11}\left(\Psi / T_{9}\right)^{1 / 3}-\left(0.22 \alpha T_{9}\right)^{2}} P_{I B}\left(\Psi, T_{9}, 2.10,3.65,0.54,-5.30,-1.98\right) \\
\quad+\Xi(2 / 3) \Sigma(0) T_{9}^{3 / 2}\left[5.54 \times 10^{-34} e^{-30.44 / T_{9}}+7.98 \times 10^{-38} e^{-4.479 / T_{9}}\right]\end{array}$ \\
\hline
\end{tabular}

The expressions for the cross sections vary with the temperature. Moreover, in the range of energies relevant for our calculation there are certain reactions that proceed through many resonances.

TABLE 5

Charged Particle Reaction Rates II

Note. $-\Theta=\Omega_{B} h^{2} T_{9}^{7 / 3} \alpha^{1 / 3} \mu^{-1 / 3}, \Psi=\mu \alpha^{2}, \Xi(b)=\Omega_{B} h^{2} \mu^{-b}$,

$\Sigma(a)=\left(\alpha / \alpha^{\mathrm{tod}}\right)\left(1+a-a\left[\alpha / \alpha^{\mathrm{tod}}\right]\right)$,

$P_{I B}\left(\Psi, T_{9}, c_{1}, c_{2}, c_{3}, c_{4}, c_{5}\right)=1+c_{1} \times 10^{-12} \Psi^{-1 / 3} T_{9}^{1 / 3}+c_{2} \times 10^{10} \Psi^{1 / 3} T_{9}^{2 / 3}+c_{3} T_{9}+c_{4} \times 10^{20} \Psi^{2 / 3} T_{9}^{4 / 3}+c_{5} \times 10^{10} \Psi^{1 / 3} T_{9}^{5 / 3}$,

$P_{L N}\left(x, d_{1}, d_{2}, d_{3}, d_{4}, d_{5}\right)=1+d_{1} x+d_{2} x^{2}+d_{3} x^{3}+d_{4} x^{4}+d_{5} x^{5}, T_{9 v}=v T_{9}+11.605$. 
TABLE 6

Neutral Particle Reactions Rates

\begin{tabular}{|c|c|}
\hline Reaction & $\begin{array}{l}\text { Reaction Rate } \\
\qquad\left(\mathrm{s}^{-1}\right)\end{array}$ \\
\hline 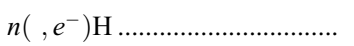 & $(16 / 60) \pi^{2} G_{\mathrm{F}}^{2} m_{e}^{5}\left[\sqrt{q^{2}-1}\left(2 q^{4}-9 q^{2}-8\right)\right]+15 q \ln \left(q+\sqrt{q^{2}-1}\right)$ \\
\hline $\mathrm{H}(n, \gamma) d$ & $40.92 \Omega_{B} h^{2} \Sigma_{\mathrm{nc}}(-1) T_{9}^{3} P_{F}\left(T_{9},-0.86,0.43\right)$ \\
\hline 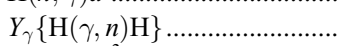 & $2.70 \times 10^{49} \Sigma_{\mathrm{nc}}(-1)\left(m_{p} m_{n} / m_{d}\right)^{3 / 2} T_{9}^{3 / 2} e^{-11.605 \epsilon_{d} / T_{9}} P_{F}\left(T_{9},-0.86,0.43\right)$ \\
\hline 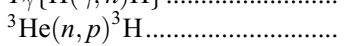 & $6.53 \times 10^{5} \Omega_{B} h^{2} T_{9}^{3} \Sigma_{\mathrm{nc}}(0.3) P_{F}\left(T_{9},-0.59,0.1832\right)$ \\
\hline${ }^{3} \mathrm{H}(p, n)^{3} \mathrm{He}$ & $6.53 \times 10^{5} \Omega_{B} h^{2} \Sigma_{\mathrm{nc}}(0.3) \Sigma_{\mathrm{nc}}(-1)\left(m_{3} m_{n} / m_{T} m_{p}\right)^{3 / 2} P_{F}\left(T_{9},-0.59,0.1832\right) e^{-11.605 Q_{6} / T_{9}} T_{9}^{3}$ \\
\hline 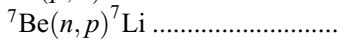 & $6.27 \times 10^{6} \Omega_{B} h^{2} \Sigma_{\mathrm{nc}}(0.2) T_{9}^{3} P_{F}\left(T_{9},-0.903,0.215\right)$ \\
\hline
\end{tabular}

In this case, we have to include an extra term in the cross section. There are two kinds of resonances single resonance and continuum resonance. In each case we use the expressions given by Fowler et al. $(1967,1975)$.

\subsubsection{Resonance Cross Sections}

In this case, the following expression from Fowler et al. (1975) provides a good fit to the cross section:

$$
\sigma(E)=\frac{\pi \hbar^{2}}{2 \mu E} \frac{\omega_{r} \Gamma_{1} \Gamma_{2}}{\left(E-E_{r}\right)^{2}+\Gamma^{2} / 4},
$$

where $\Gamma_{i}$ is the partial width for the decay of the resonant state by the reemission of $(i-1)+i, \Gamma$ is the sum over all partial widths (the partial widths are not functions of $\alpha$ ), $\omega_{r}=\left(1+\delta_{a b}\right) g_{r} / g_{a} g_{b}$ and $g_{r}=2 J_{r}+1, J_{r}$ are the spin of the resonant state, $\mu$ is in $\mathrm{kg}$, and $E_{r}$ is the resonance energy in the center of momentum system and depends on the nuclear radius. Finally, the Boltzmann cross section $\langle\sigma v\rangle$ can be calculated as

$$
\langle\sigma v\rangle=\left(\frac{2 \pi \hbar^{2}}{\mu k T}\right)^{3 / 2} \frac{(\omega \gamma)_{r}}{\hbar} e^{E_{r} / k T} \mathrm{~cm} \mathrm{~s}^{-1}
$$

where $\gamma_{r}=\left(\Gamma_{1} \Gamma_{2} / \Gamma\right)_{r}$. Here, the cross section depends on the fundamental constants through the final temperature and the resonance energy. The resonance width is also a function of the fundamental constants, but the cross section is much less sensitive to this dependence.

In a theory with massless quarks, $E_{r} \sim \Lambda_{\mathrm{OCD}}$. On the other hand, $E_{r}$ does not depend on $\alpha$. (The dependence of the temperature its analyzed in $\S 4$.) This correction is relevant for the reactions ${ }^{2} \mathrm{H}(\alpha, \gamma){ }^{6} \mathrm{Li},{ }^{6} \mathrm{Li}(p, \alpha){ }^{3} \mathrm{H},{ }^{6} \mathrm{Li}(\alpha, \gamma){ }^{10} \mathrm{Be},{ }^{7} \mathrm{Be}(p, \gamma){ }^{8} \mathrm{Be}$, and ${ }^{7} \operatorname{Li}(p, \alpha){ }^{4} \mathrm{He}$.

\subsubsection{Continuum Resonances}

When the temperature scale is of order $T_{9} \sim 1$, there are several reactions that proceed through many resonances that are separated by intervals not greater than their widths, or that overlap to form a continuum. In these cases, the cross section can be written as (Fowler et al. 1975)

$$
\sigma(E)= \begin{cases}2 \sigma(2 C) \frac{C}{E}\left(\frac{E}{C}-1\right)^{m+1 / 2} & \text { if } E \geq C \\ 0 & \text { if } E \leq C\end{cases}
$$

where $m$ is an integer or rational fraction, $C$ is the effective continuum threshold energy, and $\sigma(2 C)$ is the cross section at $E=2 C$.
After inserting this expression in the integral (21), we obtain

$$
\langle\sigma v\rangle=\Gamma(m+3 / 2) \sigma(2 C) \sqrt{\frac{32 C}{\pi \mu}}\left(\frac{k T}{C}\right)^{m} e^{-C / k T},
$$

where $\Gamma(m+3 / 2)$ is the gamma function. Here, $C$ has units of energy, so, in the chiral limit, $C \sim \Lambda_{\mathrm{QCD}}$. This correction is relevant for the reactions ${ }^{3} \mathrm{He}(d, p){ }^{4} \mathrm{He},{ }^{3} \mathrm{H}(d, n){ }^{4} \mathrm{He},{ }^{6} \mathrm{Li}(p, \alpha){ }^{3} \mathrm{H}$, and ${ }^{6} \operatorname{Li}(\alpha, \gamma){ }^{10} \mathrm{Be}$.

\subsection{Neutron Particle Reaction Rates}

In this case there is no Coulomb barrier, so the cross section cannot be written as equation (23). Following Fowler et al. (1967) we write

$$
\sigma(E)=\frac{S(E)}{v}
$$

where $v$ is the relative velocity. We consider the expression given by Fowler et al. (1967),

$$
S(E)=S(0)+\left(\frac{d S}{d \tilde{E}}\right)_{\tilde{E}=0} E^{1 / 2}+\frac{1}{2}\left(\frac{d^{2} S}{d \tilde{E}^{2}}\right)_{\tilde{E}=0} E,
$$

where $\tilde{E}=E^{1 / 2}$.

In chiral limit $\sigma \sim \Lambda_{\mathrm{QCD}}^{-2}$, and therefore

$$
S(0) \sim \Lambda_{\mathrm{QCD}}^{-2} .
$$

In this way, the expression for the reaction rates (eq. [21]) yields:

$$
\langle\sigma v\rangle=S(0)\left[1+\frac{2}{\sqrt{\pi}} \frac{S^{\prime}(0)}{S(0)}(k T)^{1 / 2}+\frac{3}{4} \frac{S^{\prime \prime}(0)}{2 S(0)} k T\right],
$$

where $d S / d E$ is in units of $\mathrm{cm}^{3} \mathrm{~s}^{-1} \mathrm{MeV}^{-1 / 2}$ and $d^{2} S / d \tilde{E}$ in units of $\mathrm{cm}^{3} \mathrm{~s}^{-1} \mathrm{MeV}^{-1}$.

For radiative emission reactions, the cross section should be multiplied by a factor $\alpha / \alpha^{\text {tod }}$. Table 6 shows some reaction rates between a neutron and a nucleus.

In some cases the reaction rates of the inverse reactions are needed. Next, we show the expressions for these reaction rates. For inverse reactions of the form $[B C A n]$, where neither $B$ nor $C$ are photons, we use the expression given by Fowler et al. (1967, 1975)

$[B C A n]=\frac{2\left(1+\delta_{B C}\right) g_{A}}{\left(1+\delta_{A n}\right) g_{B} g_{C}}\left(\frac{m_{A} m_{n}}{m_{B} m_{C}}\right)^{3 / 2} e^{-Q / k T}[A n C B] \mathrm{s}^{-1}$, 
where $Q=m_{A}+m_{n}-m_{B}-m_{C}$. In this case, the form factor $S(0)$ should be multiplied by $\alpha / \alpha^{\text {tod }}$ because of the Coulomb barrier.

For inverse reactions of the form $[\mathrm{B} \gamma \mathrm{nA}]$, we use the expression given by Fowler et al. $(1967,1975)$

$$
\begin{aligned}
Y_{\gamma}[B \gamma n A]= & \frac{g_{A} g_{n}}{\left(1+\delta_{A n}\right) g_{B}}\left(\frac{m_{A} m_{n}}{m_{B}}\right)^{3 / 2}\left(\frac{M_{U}^{2} k T}{2 \pi \hbar^{2}}\right)^{3 / 2} \\
& \times e^{-Q /(k T)}\langle\sigma v\rangle \mathrm{s}^{-1},
\end{aligned}
$$

where $Q=m_{A}+m_{n}-m_{B}, g_{n}=2 j_{n}+1=2$, and $M_{U}=1 / N_{\mathrm{A}}$.

In both cases, the additional dependence on the fundamental constants introduced by the inverse reactions proceed from the temperature (see $\S 4$ ) and the masses (see $\S 2$ ).

\subsection{Neutron Lifetime}

Neutron $\beta$ decay is one of the few reactions whose cross section can be explicitly computed from first principles in terms of the fundamental constants. It can be approximated by the one-point interaction of neutron, proton, electron, and neutrino. The reaction rate for neutron $\beta$ decay is

$$
n \rightarrow p+e^{-}+\overline{\nu_{e}} .
$$

Following Ichikawa \& Kawasaki (2002) we write the inverse of neutron lifetime as

$$
\frac{1}{\tau} \simeq G_{\mathrm{F}}^{2} \int_{0}^{P_{0}} d^{3} p_{e} d^{3} p_{\nu} \delta\left(E_{e}+E_{\nu}+m_{p}-m_{n}\right),
$$

where $G_{\mathrm{F}}$ is Fermi coupling constant, $E_{e}$ and $E_{\nu}$ are the electron and neutrino energies, respectively, and $p_{e}$ and $p_{\nu}$ are the electron and neutrino momenta. After integration we obtain

$$
\begin{aligned}
\frac{1}{\tau}= & \frac{16}{60} \pi^{2} G_{\mathrm{F}}^{2} m_{e}^{5} \\
& \times\left[\sqrt{q^{2}-1}\left(2 q^{4}-9 q^{2}-8\right)+15 q \ln \left(q+\sqrt{q^{2}-1}\right)\right] \mathrm{s}^{-1},
\end{aligned}
$$

where $m_{e}$ is the electron mass, $q=Q / m_{e}=\left(m_{n}-m_{p}\right) / m_{e}$.

In such a way, we obtain the dependence of the neutron decay rate $\tau^{-1}$ on $G_{\mathrm{F}}$ and on the mass difference (which is a function of $\alpha$ and $\Lambda_{\mathrm{QCD}}$; see $\S 2$ )

$$
\frac{\delta[n]}{[n]}=-\frac{\delta \tau}{\tau}=2 \frac{\delta G_{\mathrm{F}}}{G_{\mathrm{F}}}+6.54 \frac{\delta \Lambda_{\mathrm{QCD}}}{\Lambda_{\mathrm{QCD}}}-3.839 \frac{\delta \alpha}{\alpha} .
$$

\section{ABUNDANCES AS FUNCTIONS OF FUNDAMENTAL CONSTANTS}

In this section we calculate the abundances of light elements and their dependence on fundamental constants. First we obtain the neutron abundance until the freeze-out time of weak interaction. After this time the neutrons decay freely into protons and electrons, so their abundance only changes due to this decay.

The general form of the equations that govern the abundances of the light elements is

$$
\dot{Y}_{i}=J(t)-\Gamma(t) Y_{i},
$$

where $J(t)$ and $\Gamma(t)$ are time-dependent source and sink terms, and the dot denotes the time derivative. The time-dependent static solution of this equation is what we call following Esmailzadeh et al. (1991), the quasi-static equilibrium (QSE) solution

$$
f_{i}=\frac{J(t)}{\Gamma(t)} .
$$

To determine the formation of light nuclei we solve the following equations using only the most important reactions according to the rates of production and destruction following the criteria established by Esmailzadeh et al. (1991):

$$
\begin{aligned}
\dot{Y}_{n}= & Y_{d} Y_{d}[d d n 3]+Y_{d} Y_{T}[d T n \alpha]+Y_{p} Y_{T}[p T n 3]+Y_{d} Y_{\gamma}[d \gamma n p] \\
& -Y_{n} Y_{p}[n p d \gamma]-Y_{n} Y_{3}[n 3 T p]-Y_{n}[n] \\
\dot{Y}_{d}= & Y_{n} Y_{p}[n p d \gamma]-2 Y_{d} Y_{d}([d d p T]+[d d n 3])-Y_{d} Y_{T}[d T n \alpha] \\
& -Y_{d} Y_{3}[d 3 p \alpha]-Y_{d} Y_{\gamma}[d \gamma n p]-Y_{d} Y_{p}[d p 3 \gamma] \\
\dot{Y}_{3}= & Y_{d} Y_{p}[p d 3 \gamma]+Y_{T} Y_{p}[p T n 3]+Y_{d} Y_{d}[d d n 3] \\
& -Y_{d} Y_{3}[d 3 p \alpha]-Y_{n} Y_{3}[n 3 p T] \\
\dot{Y}_{T}= & Y_{n} Y_{3}[n 3 p T]+Y_{d} Y_{d}[d d p T]-Y_{d} Y_{T}[d T n \alpha] \\
& -Y_{p} Y_{T}[p T n 3]-Y_{p} Y_{T}[p T \gamma \alpha] \\
\dot{Y}_{6}= & Y_{d} Y_{\alpha}[d \alpha 6 \gamma]-Y_{n} Y_{6}[n 6 \alpha T]-Y_{p} Y_{6}[p 6 T \alpha] \\
\dot{Y}_{7}= & Y_{n} Y_{\alpha}[n 67 \gamma]+Y_{n} Y_{B}[n B p 7]+Y_{T} Y_{\alpha}[T \alpha 7 \gamma] \\
& -Y_{p} Y_{7}[p 7 \alpha \alpha]-Y_{n} Y_{7}[n 78 \gamma] \\
\dot{Y}_{B}= & Y_{p} Y_{6}[p 6 B \gamma]+Y_{3} Y_{\alpha}[3 \alpha B \gamma]-Y_{\gamma} Y_{B}[B \gamma 3 \alpha]-Y_{n} Y_{B}[n B p 7] \\
& -Y_{p} Y_{B}[p B \gamma 8]-Y_{d} Y_{B}[d B \alpha \alpha p] \\
\dot{Y}_{\alpha}= & Y_{d} Y_{3}[d 3 p \alpha]+Y_{n} Y_{3}[n 3 \alpha \gamma]+Y_{d} Y_{T}[d T n \alpha]+Y_{p} Y_{T}[p T \gamma \alpha],
\end{aligned}
$$

where $n$ refers to neutron, $p$ to proton, $d$ to deuterium, $T$ to tritium, 3 to ${ }^{3} \mathrm{He}, \alpha$ to ${ }^{4} \mathrm{He}, 6$ to ${ }^{6} \mathrm{Li}, 7$ to ${ }^{7} \mathrm{Li}, B$ to ${ }^{7} \mathrm{Be}, \gamma$ to the photon, $[i j k l]$ is the rate of the reaction $i+j \rightarrow k+l$, and $Y_{i}$ is the abundance of the $i$ element relative to baryons $\left(Y_{i}=n_{i} / n_{B}\right)$. In addition, these equations obey neutron number conservation,

$$
\dot{Y}_{n}+\dot{Y}_{d}+\dot{Y}_{3}+2 \dot{Y}_{T}+2 \dot{Y}_{\alpha}=-Y_{n}[n] .
$$

The method of Esmailzadeh et al. (1991) consists of calculating the different abundances between fixed points or stages. We solve equations (48)-(55) only for one element in each stage. For the other elements it is necessary to solve the quasistatic equilibrium equation using only the most important rates of production and destruction. Next, we perform the calculation of all final temperatures and abundances and all freeze-out temperatures numerically. Table 7 shows the different stages and the used equation.

The equations that describe the production of $n, \mathrm{D},{ }^{3} \mathrm{He}$, and $\mathrm{T}$ are independent to the equations for ${ }^{6} \mathrm{Li},{ }^{7} \mathrm{Li}$, and ${ }^{7} \mathrm{Be}$. Therefore, we solve the first three using the quasi-static equilibrium equation, and then use these results to calculate the other abundances.

To calculate the final abundance of light elements it is necessary to know the freeze-out temperature. The freeze-out of the production of each element happens when the most important destruction reaction rate equals the expansion rate of the universe. The dependence of the freeze-out temperatures and final temperature of each stage with the fundamental constants, will be calculated by deriving the equation that determines each temperature. Each section in this chapter discusses the calculation of abundances during a certain stage. 
TABLE 7

Stages and Equations

\begin{tabular}{|c|c|c|}
\hline Stage & Equations & Final Temperature \\
\hline 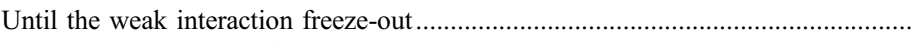 & \multirow{4}{*}{$\begin{array}{l}\dot{Y}_{n}=-2 \dot{Y}_{\alpha}-Y_{n}[n], \dot{Y}_{d}=\dot{Y}_{3}=\dot{Y}_{T}=0 \\
\dot{Y}_{n}=-2 \dot{Y}_{\alpha}, \dot{Y}_{d}=\dot{Y}_{3}=\dot{Y}_{T}=0 \\
\dot{Y}_{d}=-2 \dot{Y}_{\alpha}, \dot{Y}_{n}=\dot{Y}_{3}=\dot{Y}_{T}=0\end{array}$} & \multirow{4}{*}{$\begin{array}{l}2 \dot{Y}_{\alpha} \sim Y_{n}[n] \\
Y_{n}=Y_{d} \\
T_{9} \rightarrow 0\end{array}$} \\
\hline 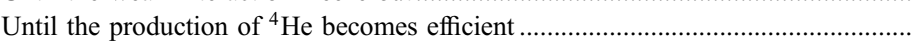 & & \\
\hline Production of deuterium dominates the rate of change of neutron abundance ........ & & \\
\hline Deuterium (final abundance) & & \\
\hline
\end{tabular}

\subsection{Neutron Abundance until the Freeze-out of Weak Interaction, $T>9.1 \times 10^{9} \mathrm{~K}$}

For the calculation of neutron abundance we follow the analysis performed by Bernstein et al. (1989). Let $\lambda_{p n}(T)$ be the rate of weak process that convert protons into neutrons and $\lambda_{n p}(T)$ the rate of weak process that convert neutrons into protons. The basic rate equation reads

$$
\frac{d X}{d t}=\lambda_{p n}(t)[1-X(t)]-\lambda_{n p}(t) X(t),
$$

where $t$ is the time, and $X$ is the ratio of the number of neutrons to the total number of baryons. After changing variables $(y=$ $\Delta m / T)$, the solution of the last equation can be written as

$$
X(y)=X_{\mathrm{eq}}(y)+\int_{0}^{y} d y^{\prime} e^{y^{\prime}}\left[X_{\mathrm{eq}}\left(y^{\prime}\right)\right]^{2} e^{K(y)-K\left(y^{\prime}\right)},
$$

where

$$
\begin{aligned}
K(y) & =b\left[\frac{4}{y^{3}}+\frac{3}{y^{2}}+\frac{1}{y}+\left(\frac{4}{y^{3}}+\frac{1}{y^{2}}\right) e^{-y}\right] \\
b & =255 \frac{M_{\mathrm{P} 1}}{\Delta m^{2} \tau} \sqrt{\frac{45}{43 \pi^{3}}} \\
X_{\mathrm{eq}}(y) & =\frac{1}{1+e^{y}},
\end{aligned}
$$

where $\tau$ is the neutron mean life and $\Delta m=m_{n}-m_{p}$. In order to obtain the asymptotic behavior, the limit $T \rightarrow 0$ or $y \rightarrow \infty$ is taken:

$$
X(y=\infty)=\int_{0}^{\infty} d y^{\prime} e^{y^{\prime}} X_{\mathrm{eq}}\left(y^{\prime}\right)^{2} e^{-K\left(y^{\prime}\right)}=0.151 .
$$

In the last equation, only $b$ depends on the fundamental constants through $\tau$ and $\Delta m$ (see $\delta \S 2$ and 3 for the dependence of these quantities on the fundamental constants). In this way, from equation (60), we obtain

$\frac{\delta X(y=\infty)}{X(y=\infty)}=-1.04 \frac{\delta G_{\mathrm{F}}}{G_{\mathrm{F}}}-2.361 \frac{\delta \Lambda_{\mathrm{QCD}}}{\Lambda_{\mathrm{QCD}}}+1.386 \frac{\delta \alpha}{\alpha}$.

\subsection{Abundances until the Production of ${ }^{4} \mathrm{He}$ Becomes Efficient, $9.1 \times 10^{9} \mathrm{~K}>T>0.93 \times 10^{9} \mathrm{~K}$}

After the freeze-out of the weak interactions, the only change in the neutron abundance is due to neutron decay. Therefore, the neutron abundance in this stage reads

$$
Y_{n}=X(y=\infty) e^{-t / \tau}=e^{-0.198 / T_{9}^{2}} .
$$

In the beginning of this stage there are no nuclei with two or more nucleons; therefore a good approximation to consider is $Y_{p} \simeq 1-Y_{n}$. However, as the universe expands, the temperature goes down and light nuclei formation begins. Therefore, at the end of this stage, this expression is no longer valid.

In order to get a consistent solution of equation (56) (Esmailzadeh et al. 1991), it is necessary to set all the rates equal to zero with the exception of the largest rate, which equals $-2 \dot{Y}_{\alpha}-Y_{n}[n]$. Thus, the equations to solve in this stage are

$$
\begin{gathered}
\dot{Y}_{n}=-2 \dot{Y}_{\alpha}-Y_{n}[n], \\
\dot{Y}_{d}=\dot{Y}_{3}=\dot{Y}_{T}=0 .
\end{gathered}
$$

\begin{tabular}{|c|c|c|}
\hline$T_{9}$ & Nucleus & Solution \\
\hline \multirow[t]{3}{*}{$9.1>T_{9}>0.93 \ldots$} & $\mathrm{D}$ & $Y_{d}=Y_{n} Y_{p}[n p d \gamma] / Y_{\gamma}[d \gamma n p]$ \\
\hline & $\mathrm{T}$ & $Y_{T}=\frac{Y_{d} Y_{p}[p d 3 \gamma]+Y_{d} Y_{d}[d d n 3]+Y_{T} Y_{p}[p \operatorname{Tn} 3]}{Y_{d}[d 3 p \alpha]+Y_{n}[n 3 p T]}$ \\
\hline & ${ }^{3} \mathrm{He}$ & $Y_{3}=\frac{Y_{n} Y_{3}[n 3 p T]+Y_{d} Y_{d}[d d p T]}{Y_{d}[d T n \alpha]+Y_{p}[p \operatorname{Tn} 3]}$ \\
\hline \multirow[t]{3}{*}{$0.93>T_{9}>0.765 \ldots \ldots \ldots \ldots \ldots$} & $\mathrm{D}$ & $Y_{d}=Y_{n} Y_{p}[n p d \gamma] / Y_{\gamma}[d \gamma n p]$ \\
\hline & $\mathrm{T}$ & $Y_{3}=Y_{d}[d d n 3] /[d 3 p \alpha]$ \\
\hline & ${ }^{3} \mathrm{He}$ & $Y_{T}=Y_{d}[d d p T] /[d T n \alpha]$ \\
\hline \multirow[t]{3}{*}{$T_{9} \rightarrow 0$} & $\mathrm{D}$ & $Y_{d}=Y_{n} Y_{p}[n p d \gamma] / Y_{\gamma}[d \gamma n p]$ \\
\hline & $\mathrm{T}$ & $Y_{3}=Y_{d}[d d n 3] /[d 3 p \alpha]$ \\
\hline & ${ }^{3} \mathrm{He}$ & $Y_{T}=Y_{d}[d d p T] /[d T n \alpha]$ \\
\hline
\end{tabular}

Table 8 shows the solutions.

When the production of ${ }^{4} \mathrm{He}$ becomes efficient, the stage ends. The final temperature is given by setting $\dot{Y}_{n}=0$ in equation (63). For this stage, we obtain $T_{9}^{f}=0.93$ and the following results:

$$
\begin{aligned}
& Y_{p}^{f}=0.76, \quad Y_{d}^{f}=4.1 \times 10^{-4}, \quad Y_{T}^{f}=2.0 \times 10^{-5}, \\
& Y_{n}^{f}=0.12, \quad Y_{3}^{f}=5.8 \times 10^{-8}, \quad Y_{\alpha}^{f}=0.06 \text {, }
\end{aligned}
$$

TABLE 8

Solutions of the Quasi-static Equilibrium Equations for Each Stage 
where $Y_{i}^{f}$ is the final abundance of each nucleus or nucleons at this stage. It follows that the abundances of $\mathrm{D}, \mathrm{T}$, and ${ }^{3} \mathrm{He}$ are negligible respect to the abundances of neutrons and ${ }^{4} \mathrm{He}$. This means

$$
\begin{aligned}
Y_{p}^{f} & =1-Y_{n}^{f}-Y_{d}^{f}-Y_{T}^{f}-2 Y_{\alpha}^{f}-2 Y_{3}^{f} \\
& \simeq 1-Y_{n}^{f}-2 Y_{\alpha}^{f}=1-2 Y_{n}^{f} .
\end{aligned}
$$

Now, in order to calculate the dependence of the final temperature with the fundamental constants for this stage, we differentiate the equation $2 \dot{Y}_{\alpha}=Y_{n}[n]$ with respect to the fundamental constants and the temperature. In such way, we obtain

$\frac{\delta T_{9}^{f}}{T_{9}^{f}}=0.068 \frac{\delta \Omega_{B} h^{2}}{\Omega_{B} h^{2}}-0.053 \frac{\delta G_{\mathrm{F}}}{G_{\mathrm{F}}}+0.063 \frac{\delta \alpha}{\alpha}+0.871 \frac{\delta \Lambda_{\mathrm{QCD}}}{\Lambda_{\mathrm{QCD}}}$,

where we also considered the dependence with the baryon fraction. Finally, the dependence of the abundance of neutrons on the fundamental constants and $\Omega_{B} h^{2}$ yields

$$
\frac{\delta Y_{n}}{Y_{n}}=0.029 \frac{\delta \Omega_{B} h^{2}}{\Omega_{B} h^{2}}-1.522 \frac{\delta G_{\mathrm{F}}}{G_{\mathrm{F}}}+2.296 \frac{\delta \alpha}{\alpha}-3.459 \frac{\delta \Lambda_{\mathrm{QCD}}}{\Lambda_{\mathrm{QCD}}} .
$$

\subsection{Final Abundance of ${ }^{4} \mathrm{He}$}

The next stage corresponds to the calculation until the rate of production of deuterium dominates over the rate of change of neutrons. However, the freeze-out temperature of ${ }^{4} \mathrm{He}(T=$ $\left.0.915 \times 10^{9} \mathrm{~K}\right)$ is lower than the final temperature of the previous stage, but higher than the final temperature of the next one. Therefore, we calculate now the final abundance of ${ }^{4} \mathrm{He}$. In this case, the neutron number conservation equation reads

$$
2 \dot{Y}_{\alpha}=Y_{n}[n]
$$

For the other nuclei the quasi-static equilibrium equation is valid (see Table 8 ). The production of ${ }^{4} \mathrm{He}$ is dominated by $[d T n \alpha]$ and $[p T \gamma \alpha]$ :

$$
\begin{aligned}
\dot{Y}_{\alpha} & =Y_{d} Y_{T}[d T n \alpha]+Y_{p} Y_{T}[p T \gamma \alpha] \\
& =\left(Y_{n} Y_{p} \frac{[n p d \gamma]}{Y_{\gamma}[d \gamma n p]}\right)^{2}[d d p T]
\end{aligned}
$$

After solving equation (68) numerically for $T_{9}$, we obtain $T_{9}^{\alpha}=0.915$ and $Y_{\alpha}^{f}=2 Y_{n}=0.238$. When the rate of ${ }^{4} \mathrm{He}$ production equals the rate of neutron destruction, there are no more neutrons that can form ${ }^{4} \mathrm{He}$. Since this happens earlier than the usual freeze-out time, we use equation (68) to calculate the freeze-out temperature. In this way, the dependence of the freeze-out temperature on the fundamental constants and $\Omega_{B} h^{2}$ yields

$\frac{\delta T_{9}^{\alpha}}{T_{9}^{\alpha}}=0.061 \frac{\delta \Omega_{B} h^{2}}{\Omega_{B} h^{2}}-0.052 \frac{\delta G_{\mathrm{F}}}{G_{\mathrm{F}}}+0.063 \frac{\delta \alpha}{\alpha}+0.869 \frac{\delta \Lambda_{\mathrm{QCD}}}{\Lambda_{\mathrm{QCD}}}$.
Finally, since $Y_{\alpha}^{c}=2 Y_{n}$, we can express the variation of the final abundance of ${ }^{4} \mathrm{He}$ as a function of fundamental constants and $\Omega_{B} h^{2}$,

$$
\frac{\delta Y_{\alpha}^{c}}{Y_{\alpha}^{c}}=0.029 \frac{\delta \Omega_{B} h^{2}}{\Omega_{B} h^{2}}-1.538 \frac{\delta G_{\mathrm{F}}}{G_{\mathrm{F}}}+2.324 \frac{\delta \alpha}{\alpha}-3.496 \frac{\delta \Lambda_{\mathrm{QCD}}}{\Lambda_{\mathrm{QCD}}} .
$$

\subsection{Neutron Cooking, $0.93 \times 10^{9} \mathrm{~K}>T>0.765 \times 10^{9} \mathrm{~K}$}

In this section we calculate the deuterium abundance as long as the rate of change of neutron production dominates the deuterium rate. This is valid until the production rate of deuterium dominates the rate of change of neutrons, so this stage is over when $Y_{n}=Y_{d}$. In this stage, the neutron number conservation equation reads

$$
\dot{Y}_{n}=-2 \dot{Y}_{\alpha}
$$

For D, T, and ${ }^{3} \mathrm{He}$, we solve the quasi-static equilibrium equations. The solutions are shown in Table 8 . For ${ }^{4} \mathrm{He}$ we solve the complete equation, but considering only the largest production term $Y_{d} Y_{T}[d T n \alpha]$. Inserting all these solutions in equation (72), we obtain

$$
\dot{Y}_{n}=-2\left(Y_{n} Y_{p} \frac{[n p d \gamma]}{Y_{\gamma}[d \gamma n p]}\right)^{2}[d d p T]
$$

where the initial condition is given by the final values of the previous stage, $Y_{n}^{0}=0.12$ and $T_{9}^{0}=0.93$. We can write the solution to the last equation as

$$
Y_{n}=\left(\frac{1}{Y_{n}^{0}}+2 \int_{t_{\text {init }}}^{t}\left(Y_{p} \frac{[n p d \gamma]}{Y_{\gamma}[d \gamma n p]}\right)^{2}[d d p T] d t\right)^{-1}
$$

After changing the integration variable to $T_{9}$, we integrate numerically as a function of temperature. We also compute the final temperature of this stage using the condition

$$
Y_{n}=Y_{d}
$$

We obtain

$$
T_{9}^{f}=0.765, \quad Y_{n}=6.4 \times 10^{-4}=Y_{d} .
$$

From equation (75) we obtain the dependence of the final temperature of this stage with respect to the fundamental constants and $\Omega_{B} h^{2}$,

$\frac{\delta T_{9}^{f}}{T_{9}^{f}}=0.031 \frac{\delta \Omega_{B} h^{2}}{\Omega_{B} h^{2}}+0.015 \frac{\delta G_{\mathrm{F}}}{G_{\mathrm{F}}}-0.023 \frac{\delta \alpha}{\alpha}+1.034 \frac{\delta \Lambda_{\mathrm{QCD}}}{\Lambda_{\mathrm{QCD}}}$.

Finally, the dependence of the final neutron and deuterium abundance can be obtained from equation (74):

$$
\begin{aligned}
\frac{\delta Y_{d}}{Y_{d}}=\frac{\delta Y_{n}}{Y_{n}}= & -1.099 \frac{\delta \Omega_{B} h^{2}}{\Omega_{B} h^{2}}-0.058 \frac{\delta G_{\mathrm{F}}}{G_{\mathrm{F}}} \\
& +1.871 \frac{\delta \alpha}{\alpha}-0.488 \frac{\delta \Lambda_{\mathrm{QCD}}}{\Lambda_{\mathrm{QCD}}} .
\end{aligned}
$$


TABLE 9

Quasi-static Equilibrium Solutions

\begin{tabular}{|c|c|}
\hline Nucleus & Quasi-static Equilibrium Solutions \\
\hline${ }^{3} \mathrm{He}$ & $Y_{3}=Y_{d}[d d n 3] /[d 3 p \alpha]$ \\
\hline $\mathrm{T}$. & $Y_{T}=Y_{d}[d d p T] /[d T n \alpha]$ \\
\hline ................ & $Y_{6}=Y_{d} Y_{\alpha}[d \alpha 6 \gamma] / Y_{p}[p 6 T \alpha]$ \\
\hline . & $Y_{B}=Y_{3} Y_{\alpha}[3 \alpha B \gamma] / Y_{n}[n B p 7]$ \\
\hline${ }^{7} \mathrm{Li} \ldots \ldots$. & $Y_{7}=\left(Y_{n} Y_{B}[n B p 7]+Y_{T} Y_{\alpha}[T \alpha 7 \gamma]\right) / Y_{p}[p 7 \alpha \alpha]$ \\
\hline
\end{tabular}

\subsection{Deuterium Cooking, $T \rightarrow 0$}

For temperatures lower than $T_{9}=0.765$, the largest production rate corresponds to deuterium. Therefore, we set all other derivatives to zero in equation (56). Since the largest term for deuterium destruction is tritium production, the equation to solve is

$$
\dot{Y}_{d}=-2 Y_{d} Y_{d}[d d p T]
$$

with the initial condition $Y_{d}^{0}=6.4 \times 10^{-4}$ on $T_{9}^{0}=0.765$. Since this equation has the same form as equation (73), the solution reads

$$
Y_{d}=\left(\frac{1}{Y_{d}^{0}}+2 \int_{t_{\mathrm{init}}}^{t}[d d p T] d t\right)^{-1}
$$

In order to calculate the deuterium final abundance we consider the limit $T \rightarrow 0(t \rightarrow \infty)$. We numerically obtain the deuterium final abundance $Y_{d}^{f}=2.410 \times 10^{-5}$. On the other hand, the dependence of the deuterium final abundance on the fundamental constants and $\Omega_{B} h^{2}$ can be calculated by deriving equation (80):

$\frac{\delta Y_{d}^{c}}{Y_{d}^{c}}=-1.072 \frac{\delta \Omega_{B} h^{2}}{\Omega_{B} h^{2}}-0.036 \frac{\delta G_{\mathrm{F}}}{G_{\mathrm{F}}}+2.320 \frac{\delta \alpha}{\alpha}+0.596 \frac{\delta \Lambda_{\mathrm{QCD}}}{\Lambda_{\mathrm{QCD}}}$.

\subsection{Final Abundances}

Here we calculate the freeze-out temperature and final abundances of ${ }^{3} \mathrm{He}, \mathrm{T},{ }^{6} \mathrm{Li},{ }^{7} \mathrm{Be}$, and ${ }^{7} \mathrm{Li}$ and the dependence of these quantities with the fundamental constants. In order to calculate any light element abundance it is necessary to solve the quasi-static equilibrium equation,

$$
\dot{Y}_{i}=0 .
$$

\begin{tabular}{|c|c|c|c|c|c|}
\hline$Y_{i}^{f}$ & Abundance & $A$ & $B$ & $C$ & $D$ \\
\hline${ }^{2} \mathrm{H} \ldots$. & $2.741 \times 10^{-5}$ & -0.036 & 2.320 & 0.596 & -1.072 \\
\hline${ }^{3} \mathrm{He}$ & $6.95 \times 10^{-6}$ & -0.051 & 0.983 & 0.999 & -1.102 \\
\hline${ }^{3} \mathrm{H} \ldots \ldots \ldots \ldots$ & $1.21 \times 10^{-7}$ & -0.041 & 0.252 & 0.941 & -1.083 \\
\hline${ }^{4} \mathrm{He}$ & 0.238 & -1.538 & 2.323 & -3.497 & 0.029 \\
\hline${ }^{6} \mathrm{Li}$ & $5.7 \times 10^{-14}$ & -2.061 & 7.414 & -3.462 & -1.047 \\
\hline${ }^{7} \mathrm{Be}$ & $5.60 \times 10^{-10}$ & -0.172 & -9.450 & -1.038 & 2.209 \\
\hline${ }^{7} \mathrm{Li}$ & $2.36 \times 10^{-10}$ & -0.720 & 1.824 & -3.411 & 0.068 \\
\hline
\end{tabular}

TABLE 11

Abundances and Their Dependence on Fundamental Constants

We solve these equations considering only the most relevant reactions. In Table 9 we show the quasi-static equilibrium solutions.

In order to compute the freeze-out temperature, we set the largest rate of destruction $\Gamma$ of each equation that governs the abundance of the light elements equal to the universe expansion rate $H$,

$$
\Gamma=H=\frac{1}{356} T_{9}^{2} \mathrm{~s}^{-1}
$$

Table 10 shows the different freeze-out temperatures and their dependence on fundamental constants, which are calculated deriving the previous equation. Using the freeze-out temperature we calculate the final abundance of the different nuclei and their dependence on the fundamental constants and $\Omega_{B} h^{2}$. In Table 11 we show these results.

\section{RESULTS AND DISCUSSION}

In this section we compare the theoretical predictions of the abundances of the light elements, obtained in the last section, to observational data.

In $\S 4$ we obtained seven equations of the form

$$
\frac{\delta Y_{i}^{f}}{Y_{i}^{f}}=A_{i} \frac{\delta G_{\mathrm{F}}}{G_{\mathrm{F}}}+B_{i} \frac{\delta \alpha}{\alpha}+C_{i} \frac{\delta \Lambda_{\mathrm{QCD}}}{\Lambda_{\mathrm{QCD}}}+D_{i} \frac{\delta \Omega_{B} h^{2}}{\Omega_{B} h^{2}},
$$

where $A_{i}, B_{i}, C_{i}$, and $D_{i}$ are constant coefficients (see Table 11), $\delta Y_{i} / Y_{i}=\left(Y_{i}^{\text {obs }}-Y_{i}^{\mathrm{SBBN}}\right) / Y_{i}^{\mathrm{SBBN}} ;$ and $Y_{i}^{\mathrm{SBBN}}$ and $Y_{i}^{\text {obs }}$ are the theoretical (see Table 12) and observed abundances, respectively.

However, independent observational data are only available for the abundances of $\mathrm{D},{ }^{3} \mathrm{He},{ }^{4} \mathrm{He}$, and ${ }^{7} \mathrm{Li}$. In Table 13 we show the independent data we consider in this work. For a recent review of all observational available data on primordial abun-

\begin{tabular}{|c|c|c|c|c|c|c|}
\hline Nucleus & Equation & $T_{9}^{\text {freeze-out }}$ & $W$ & $R$ & $T$ & $J$ \\
\hline${ }^{3} \mathrm{He}$. & $Y_{d}[d 3 p \alpha]=H$ & 0.403 & 0.008 & -0.510 & 1.168 & 0.016 \\
\hline .............. & $Y_{d}[d T n \alpha]=H$ & 0.105 & 0.009 & 0.122 & 1.181 & 0.018 \\
\hline${ }^{6} \mathrm{Li} \ldots .$. & $Y_{p}[p 63 \alpha]=H$ & 0.069 & -0.076 & 1.962 & 1.118 & -0.156 \\
\hline${ }^{7} \mathrm{Be}$. & $Y_{n}[n B p 7]=H$ & 0.319 & 0.217 & -0.712 & 1.39 & 0.350 \\
\hline ................ & $Y_{p}[p 7 \alpha \alpha]=H$ & 0.185 & -0.088 & 1.692 & 0.946 & -0.182 \\
\hline
\end{tabular}
dances, see Eidelman et al. (2004). On the other hand, recent papers (Coc et al. 2004a; Cyburt 2004) have called attention to

TABLE 10

Freeze-out Temperature and Its Dependence on Fundamental Constants

Note. $-\delta T_{9}^{i} / T_{9}^{i}=W\left(\delta G_{\mathrm{F}} / G_{\mathrm{F}}\right)+R(\delta \alpha / \alpha)+T\left(\delta \Lambda_{\mathrm{QCD}} / \Lambda_{\mathrm{QCD}}\right)+J\left(\delta \Omega_{B} h^{2} / \Omega_{B} h^{2}\right)$. 
TABLE 12

Theoretical Abundances in the Standard Model: WMAP Estimate, $\Omega_{B} h^{2}=0.0224$

\begin{tabular}{|c|c|}
\hline Nucleus & $Y_{i}^{\mathrm{SBBN}} \pm \delta Y_{i}^{\mathrm{SBBN}}$ \\
\hline${ }^{2} \mathrm{H} \ldots$ & $(2.51 \pm 0.37) \times 10^{-5}$ \\
\hline${ }^{3} \mathrm{He} \ldots$ & $(1.05 \pm 0.15) \times 10^{-5}$ \\
\hline${ }^{4} \mathrm{He} . .$. & $0.2483 \pm 0.0012$ \\
\hline${ }^{7} \mathrm{Li}$ & $(5.0 \pm 0.3) \times 10^{-10}$ \\
\hline
\end{tabular}

the errors introduced by the values of the cross sections involved in the calculation of the abundances. Cyburt (2004) has also analyzed the propagation through the theoretical abundances, yielding a "theoretical" percent error of $5 \%$. In the original work of Esmailzadeh et al. (1991), the error introduced by the semianalytical method is estimated to be of order 5\%. Therefore, in order to solve equation system (84), we will add to the errors of Table 13 an error of order $10 \%$.

First we perform a test to check the consistency of the data (Riveros \& Vucetich 1986). For each group of data $\left(Y_{i}\right)$ belonging to the same abundance, we calculate the weighted averaged value $\bar{Y}$ and its corresponding error $\sigma_{i}$. Then we compute

$$
\chi^{2}=\sum_{i} \frac{\left(Y_{i}-\bar{Y}\right)^{2}}{\sigma_{i}^{2}} .
$$

If the errors are Gaussian distributed, the expected value of $\chi^{2}$ is $k-1$, where $k$ is the number of data points in each group. Furthermore, the corresponding ideogram method $\operatorname{plot}^{5}$ of each group of data ${ }^{6}$ should be a Gaussian. It follows from Figure 1 and from the calculation of $\chi^{2}$ that $\mathrm{D}$ and ${ }^{4} \mathrm{He}$ data are not Gaussian distributed. However, since $\Theta=\left[\chi^{2} /(k-1)\right]^{1 / 2}$ is not that much greater than 1 , we can use the data by increasing the observational error by a factor $\Theta$. The values of $\Theta$ are 2.4 for $D, 2.33$ for ${ }^{4} \mathrm{He}$.

We assume that any difference between the theoretical abundance and the observational abundance is due to the variation of fundamental constants. In such a way, the solution of

\footnotetext{
5 See http://rkb.home.cern.ch/rkb/AN16pp/node126.html.

${ }^{6}$ From the Particle Data Group: see http://pdg.lbl.gov/.
}

TABLE 13

Observational Abundances Used in This Work

\begin{tabular}{|c|c|c|}
\hline Nucleus & $Y_{i}^{\mathrm{obs}} \pm \delta Y_{i}^{\mathrm{obs}}$ & Reference \\
\hline \multirow[t]{6}{*}{$\mathrm{D}$} & $(1.65 \pm 0.35) \times 10^{-5}$ & Pettini \& Bowen (2001) \\
\hline & $(2.54 \pm 0.23) \times 10^{-5}$ & O’Meara et al. (2001) \\
\hline & $\left(2.42_{-0.25}^{+0.35}\right) \times 10^{-5}$ & Kirkman et al. (2003) \\
\hline & $(3.25 \pm 0.3) \times 10^{-5}$ & Burles \& Tytler (1998a) \\
\hline & $\left(3.98_{-0.67}^{+0.59}\right) \times 10^{-5}$ & Burles \& Tytler (1998b) \\
\hline & $\left(1.6_{-0.30}^{+0.25}\right) \times 10^{-5}$ & Crighton et al. (2004) \\
\hline${ }^{3} \mathrm{He} .$. & $(1.1 \pm 0.2) \times 10^{-5}$ & Bania et al. (2002) \\
\hline \multirow[t]{4}{*}{ 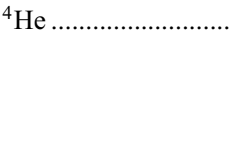 } & $0.244 \pm 0.002$ & Izotov \& Thuan (1998) \\
\hline & $0.243 \pm 0.003$ & Izotov et al. (1997) \\
\hline & $0.2345 \pm 0.0026$ & Peimbert et al. (2000) \\
\hline & $0.232 \pm 0.003$ & Olive \& Steigman (1995) \\
\hline \multirow[t]{4}{*}{${ }^{7} \mathrm{Li}$} & $\left(1.23_{-0.32}^{+0.68}\right) \times 10^{-10}$ & Ryan et al. (2000) \\
\hline & $\left(1.58_{-0.20}^{+0.24}\right) \times 10^{-10}$ & Bonifacio et al. (1997) \\
\hline & $(1.73 \pm 0.05) \times 10^{-10}$ & Bonifacio \& Molaro (1997) \\
\hline & $\left(2.19_{-0.26}^{+0.30}\right) \times 10^{-10}$ & Bonifacio et al. (2002) \\
\hline
\end{tabular}
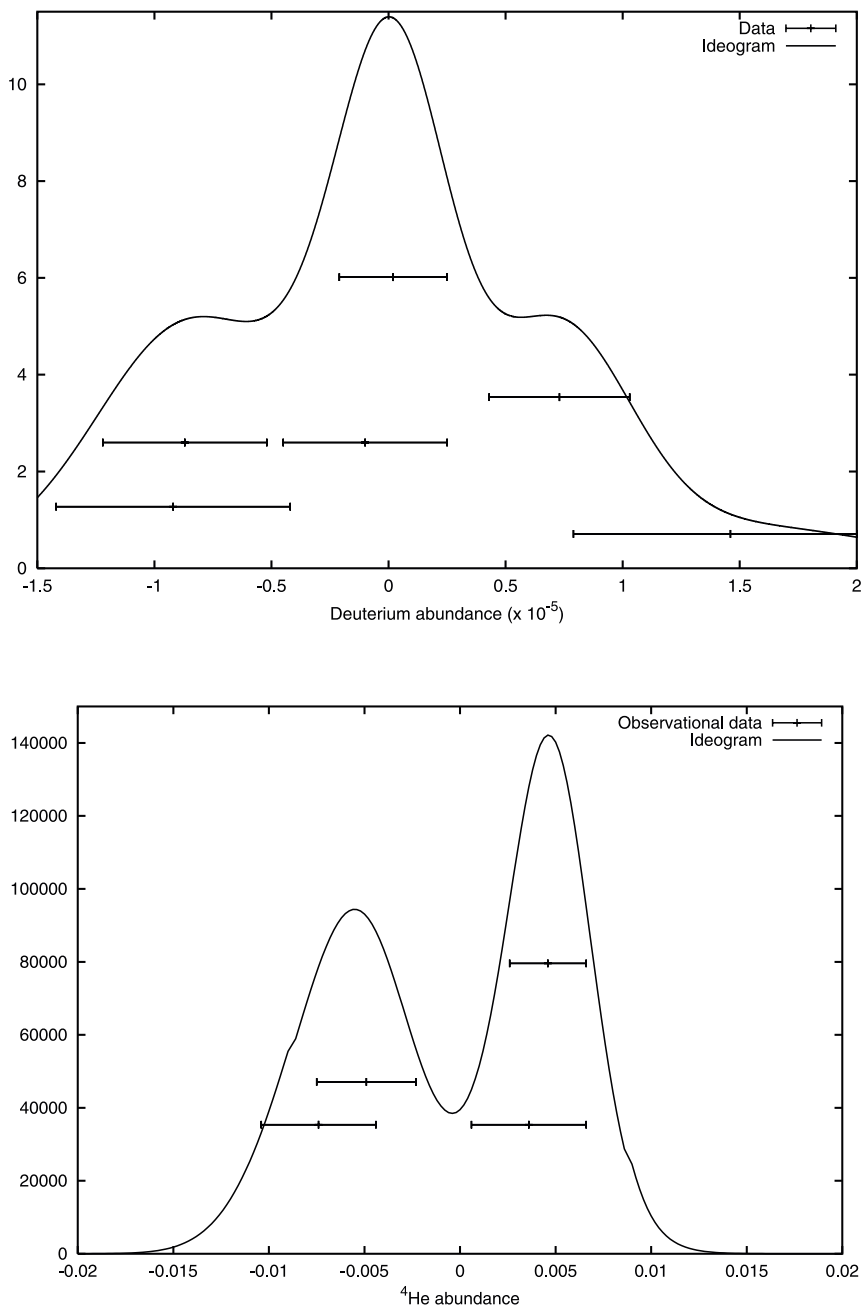

FIG. 1.-Ideograms for D and ${ }^{4} \mathrm{He}$.

equation system (84) gives a constraint to this variation. The solution is given by (Arley \& Buch 1968)

$$
\frac{\delta \alpha_{i}}{\alpha_{i}}=\left[\left(B^{t} P B\right)^{-1} B^{t} P \delta\right]_{i} \pm \sqrt{\left[\left(B^{t} P B\right)^{-1}\right]_{i i}} \mathbf{s},
$$

where $B$ is the $n \times 4$ matrix, $n$ is the number of observational data points,

$$
B=\left(\begin{array}{cccc}
A_{1} & B_{1} & C_{1} & D_{1} \\
A_{2} & B_{2} & C_{2} & D_{2} \\
\vdots & \vdots & \vdots & \vdots \\
A_{n} & B_{n} & C_{n} & D_{n}
\end{array}\right),
$$

TABLE 14

\begin{tabular}{|c|c|c|c|c|}
\hline \multirow[b]{2}{*}{ PARAMETER } & \multicolumn{2}{|c|}{ All Data } & \multicolumn{2}{|c|}{ All Data ExcePt ${ }^{7} \mathrm{Li}$} \\
\hline & Value & $\sigma$ & Value & $\sigma$ \\
\hline$\delta G_{\mathrm{F}} / G_{\mathrm{F}} .$. & -0.886 & 0.053 & -0.257 & 0.659 \\
\hline ................... & -0.136 & 0.041 & -0.054 & 0.097 \\
\hline$\delta \Lambda_{\mathrm{QCD}} / \Lambda_{\mathrm{QCD}} \ldots \ldots \ldots \ldots \ldots \ldots \ldots \ldots \ldots \ldots \ldots \ldots \ldots \ldots \ldots$ & 0.309 & 0.023 & 0.087 & 0.233 \\
\hline
\end{tabular}

Constraints on the Variation of Fundamental Constants $\left(\Omega_{B} h^{2}=0.0224\right)$ 
TABLE 15

Constraints on the Variation of $\alpha\left(\Omega_{B} h^{2}=0.0224\right)$

\begin{tabular}{|c|c|c|}
\hline \multirow[b]{2}{*}{ Source } & \multicolumn{2}{|c|}{$\delta \alpha / \alpha$} \\
\hline & Value & $\sigma$ \\
\hline All data......... & -0.041 & 0.024 \\
\hline All data but ${ }^{7} \mathrm{Li} \ldots$ & -0.015 & 0.005 \\
\hline
\end{tabular}

TABLE 17

Constraints on the Variation of $\alpha$ Using Two Independent Estimates for the Baryon Fraction

\begin{tabular}{|c|c|c|}
\hline \multirow[b]{2}{*}{ Source } & \multicolumn{2}{|c|}{$\delta \alpha / \alpha$} \\
\hline & Value & $\sigma$ \\
\hline All data.. & -0.086 & 0.034 \\
\hline All data except ${ }^{7} \mathrm{Li} .$. & -0.015 & 0.005 \\
\hline
\end{tabular}

$\delta$ is the $n \times 1$ matrix

$$
\delta=\left(\begin{array}{c}
\frac{\delta Y_{1}}{Y_{1}} \\
\frac{\delta Y_{2}}{Y_{2}} \\
\vdots \\
\frac{\delta Y_{n}}{Y_{n}}
\end{array}\right)
$$

and $P$ is the $n \times n$ matrix of weight

$$
P=\left(\begin{array}{cccc}
p_{1} & 0 & \ldots & 0 \\
0 & p_{2} & \ldots & 0 \\
\vdots & \vdots & \ddots & \vdots \\
0 & 0 & \ldots & p_{n}
\end{array}\right)
$$

where $p_{i}=1 / \sigma_{i}^{2}$ and $\sigma_{i}$ are the observational errors.

The most accurate estimation of $\Omega_{B} h^{2}$ arrives from constraining parameters with data from the CMB provided by $W M A P$ (Spergel et al. 2003). Fixing the baryon fraction with the $W M A P$ value (i.e., setting $D_{i}=0$ ), the results of solving the equation system (84) with all data listed in Table 13 are shown in Table 14. These results are consistent with variation of the fundamental constants to within $3 \sigma$. On the other hand, the results considering only variation of the fine-structure constant are shown in Table 15. These results are consistent with no variation of $\alpha$ within $3 \sigma$. In order to rule out any systematic error in the data, we computed the solution of equation system (84) again, but excluded one group of data each time. Again, the results are consistent with variation of the fundamental constants in all cases but in the case in whichthe ${ }^{7} \mathrm{Li}$ data were excluded (see Tables 14 and 15).

Even though the WMAP estimate of the baryon density is the most accurate one, it is still affected by degeneracies with other cosmological parameters (Spergel et al. 2003). Therefore, we added an independent estimation of $\Omega_{B} h^{2}$ in our analysis. In the Appendix we use data from X-ray measurements, galaxy sur-

TABLE 16

Constraints on the Variation of the Fundamental Constants Using Two

\begin{tabular}{|c|c|c|c|c|}
\hline \multirow[b]{2}{*}{ Parameter } & \multicolumn{2}{|c|}{ All Data } & \multicolumn{2}{|c|}{ All Data Except ${ }^{7} \mathrm{Li}$} \\
\hline & Value & $\sigma$ & Value & $\sigma$ \\
\hline$\delta \Omega_{B} h^{2} / \Omega_{B} h^{2} \ldots$ & 0.004 & 0.036 & 0.0005 & 0.039 \\
\hline$\delta G_{\mathrm{F}} / G_{\mathrm{F}} \ldots \ldots$ & -0.886 & 0.050 & -0.258 & 0.64 \\
\hline$\delta \alpha / \alpha \ldots \ldots$ & -0.134 & 0.044 & -0.053 & 0.095 \\
\hline$\delta \Lambda_{\mathrm{QCD}} / \Lambda_{\mathrm{QCD}}$ & 0.310 & 0.023 & 0.087 & 0.229 \\
\hline
\end{tabular}
InDEPENDENT Estimates For the Baryon Fraction veys, and Cepheids calibration in order to get an independent value of the baryon density. Furthermore, we computed again the results of $\S \S 3$ and 4, changing the value of $\Omega_{B} h^{2}$ to 0.0223 . This value is the weighted mean value between the WMAP estimate and the value of Appendix A. However, we found no difference in the value of the coefficients of the variation of fundamental constants and $\Omega_{B} h^{2}$. The results obtained solving equation system (84) including both estimates for the baryon fraction (i. e., $D_{i} \neq 0$ ) show no significant difference with respect to the case where only the WMAP value was considered (see Tables 16 and 17). Furthermore, in order to check for consistency of our method, we again solved equation system (84) allowing only for variation of $\Omega_{B} h^{2}$ with respect to the weighed mean value (i.e., $A_{i}=B_{i}=C_{i}=0$ ). These results are shown in Table 18. On the other hand, in order to learn about the degeneracies of the fundamental constants within the BBN model, we computed the correlation coefficients from the error matrix. We find that there is high correlation between $\alpha$ and $\Lambda_{\mathrm{QCD}}, \alpha$ and $G_{\mathrm{F}}$, and $\Lambda_{\mathrm{QCD}}$ and $G_{\mathrm{F}}$, while the correlation between other pairs of parameters is not significant.

In order to understand the discrepancy of the results obtained with and without the ${ }^{7} \mathrm{Li}$ data, we computed the relative residuals (Arley \& Buch 1968) and their respective theoretical and empirical probability in both cases. Figure 2 shows that in the case where both the variation of the fundamental constants and the deviation of $\Omega_{B} h^{2}$ from the WMAP estimate is considered, the theoretical and empirical probability distributions are very similar, while in the case where only the deviation of $\Omega_{B} h^{2}$ is considered, there is slight difference between the empirical probabilities (both with all data and excluding ${ }^{7} \mathrm{Li}$ data) and the theoretical probability. Including the variation of fundamental constants gives more degrees of freedom to equation system (84). Therefore, we suspect that the possible unreported systematic uncertainties "hide" under the variation of the fundamental constants. We also performed a Kolmogorov-Smirnov (K-S) test, in order to check the goodness of our fit. For the results obtained considering variation of all constants and $\Omega_{B} h^{2}$, we obtain a probability of $21 \%$ to obtain a worse fit, while excluding the ${ }^{7} \mathrm{Li}$ data the probability lowers to $11 \%$. On the other hand, if we only consider the deviation of $\Omega_{B} h^{2}$ with respect to the WMAP data, we obtain a probability of $99 \%$ for all data, while excluding the ${ }^{7} \mathrm{Li}$ data gives a $49 \%$ probability to

\section{TABLE 18}

Constraints on the Deviations of $\Omega_{B} h^{2}$ with Respect

\begin{tabular}{|c|c|c|}
\hline \multirow[b]{2}{*}{ SOURCE } & \multicolumn{2}{|c|}{$\delta \Omega_{B} h^{2} / \Omega_{B} h^{2}$} \\
\hline & Value & $\sigma$ \\
\hline All data.. & -0.085 & 0.294 \\
\hline All data except ${ }^{7} \mathrm{Li}$ & -0.014 & 0.054 \\
\hline
\end{tabular}
to the Mean Value Considered in This Work (0.0223) 

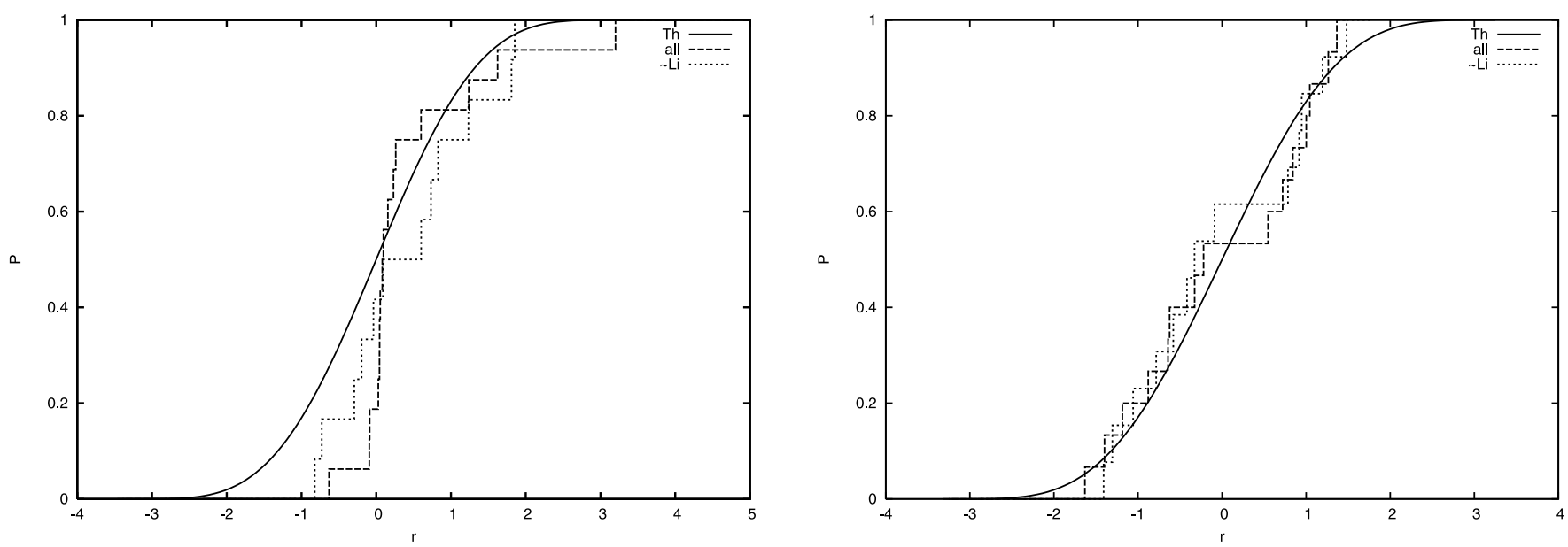

FIG. 2.-Solid line shows the theoretical probability of the residuals; dashed line shows the empirical probability computed with all data; dotted line shows the empirical probability computed with all data except ${ }^{7} \mathrm{Li}$. Left: Only deviation of $\Omega_{B} h^{2}$ with respect to its mean value is considered. Right: variation of all constants and deviation of $\Omega_{B} h^{2}$ from the mean value is considered.

get a worse fit. However, we consider the results of the K-S test only indicative, since even though the data considered are independent the residuals are not.

We mentioned in the introduction the disagreement between the ${ }^{7} \mathrm{Li}$ observational abundances with the $\mathrm{D}$ observational abundance and WMAP estimate of the baryon density. Richard et al. (2005) claim that a better understanding of turbulent transport in the stars is necessary to understand this discrepancy. Moreover, Meléndez \& Ramírez (2004) have reanalyzed the ${ }^{7} \mathrm{Li}$ data with an improved infrared flux method temperature scale, obtaining values that are marginally consistent with the WMAP estimate. However, solving equation system (84) with the ${ }^{7} \mathrm{Li}$ abundance taken from their work does not change our results in a significant way.
We adopt the conservative criterion that columns (3) and (4) of Tables 14 and 16 are the constraints on the variation of the constants we obtain with the method and hypothesis described in this paper. We also consider that more observations of ${ }^{7} \mathrm{Li}$ are needed in order to arrive to stronger conclusions. However, if the all data are correct, this analysis shows that varying coupling constants may solve the concordance problem between $\mathrm{BBN}$ and CMB. Our results are consistent to within $2 \sigma$ with the analysis performed by Ichikawa \& Kawasaki (2004), using only the variation of $\alpha$ and a nonstandard expansion rate.

H. V. is partially supported by project $42026-\mathrm{F}$ of CONACyT, Mexico, and project G11/G071, UNLP.

\section{APPENDIX}

In this appendix, we combine independent astronomical data in order to obtain an independent estimation of the baryon density. From measurements of hot gas in clusters it is possible to obtain an estimate of $\left(\Omega_{B} / \Omega_{m}\right) h^{3 / 2}$.

Ettori (2003) has called attention to the fact that the contribution from baryons in galaxies and "exotic sources" like intergalactic stars and baryonic dark matter are not considered in the results obtained from measurements of hot gas in clusters. Furthermore, Donahue et al. (2003) have estimated the contribution from the galaxies as $f_{\text {gal }}=0.15 h^{3 / 2} f_{\text {gas }}$ while the "exotic" contribution has been estimated in $f_{\text {exotic }}=0.3 f_{\text {gal }}$ (Ettori 2003). Therefore, we add to the estimation of the baryon fraction found by Donahue et al. (2003) the contribution from galaxies, yielding the value

$$
\frac{\Omega_{B}}{\Omega_{m}} h^{3 / 2}=0.0737 \pm 0.0143 .
$$

The values of the other estimates (Majerowicz et al. 2002; Castillo-Morales \& Schindler 2003) are contained within the error in this estimation.

On the other hand, $\Omega_{m} h$ has been estimated from large redshift galaxy surveys like Sloan Digital Sky Survey (Pope et al. 2004) and 2dF Galaxy Redshift Survey (Percival et al. 2001), while the most stringent bound on the Hubble constant follows from Cepheid

TABLE 19

Observational Data Used to Estimate the Baryon Density

\begin{tabular}{|c|c|c|}
\hline Parameter & Value & Source \\
\hline \multirow{3}{*}{$\left(\Omega_{B} / \Omega_{m}\right) h^{3 / 2}$} & $0.067 \pm 0.03$ & Donahue et al. (2003) \\
\hline & $0.073 \pm 0.013$ & Majerowicz et al. (2002) \\
\hline & $0.056 \pm 0.007$ & Castillo-Morales \& Schindler (2003) \\
\hline \multirow[t]{2}{*}{$\Omega_{m} h \ldots \ldots \ldots \ldots \ldots \ldots \ldots \ldots \ldots \ldots \ldots \ldots \ldots \ldots \ldots \ldots$} & $0.20 \pm 0.03$ & Percival et al. (2001) \\
\hline & $0.207 \pm 0.030$ & Pope et al. (2004) \\
\hline ................ & $0.72 \pm 0.08$ & Freedman et al. (2001) \\
\hline
\end{tabular}


calibration (Freedman et al. 2001). Combining all these data (see Table 19), and after propagating errors, we obtain a value for the baryon density of

$$
\Omega_{B} h^{2}=0.017 \pm 0.007 \text {. }
$$

This value is less accurate that the estimation done with the data of WMAP (Spergel et al. 2003),

$$
\Omega_{B} h^{2}=0.0224 \pm 0.0009,
$$

but we will consider it in order to have an independent data of this quantity.

REFERENCES

Anchordoqui, L. A., Torres, D. F., \& Vucetich, H. 1996, Phys. Lett. A, 222, 43 Arley, N., \& Buch, R. 1968, Introduction to the Theory of Probability and Statistics (New York: Wiley)

Avelino, P. P., Martins, C. J. A. P., Rocha, G., \& Viana, P. 2000, Phys. Rev. D, 62,123508

Bahcall, J. N., Steinhardt, C. L., \& Schlegel, D. 2004, ApJ, 600, 520

Bania, T. M., Rood, R. T., \& Balser, D. S. 2002, Nature, 415, 54

Barr, S. M., \& Mohapatra, P. K. 1988, Phys. Rev. D, 38, 3011

Barrow, J. D., Sandvik, H. B., \& Magueijo, J. 2002, Phys. Rev. D, 65, 063504

Battye, R. A., Crittenden, R., \& Weller, J. 2001, Phys. Rev. D, 63, 043505

Beane, S. R., \& Savage, M. J. 2003a, Nucl. Phys. A, 717, 91

2003b, Nucl. Phys. A, 713, 148

Bekenstein, J. D. 1982, Phys. Rev. D, 25, 1527

Bergström, L., Iguri, S., \& Rubinstein, H. 1999, Phys. Rev. D, 60, 45005

Bernstein, J., Brown, L. S., \& Feinberg, G. 1989, Rev. Mod. Phys., 61, 25

Bonifacio, P., \& Molaro, P. 1997, MNRAS, 285, 847

Bonifacio, P., Molaro, P., \& Pasquini, L. 1997, MNRAS, 292, L1

Bonifacio, P., et al. 2002, A\&A, 390, 91

Brax, P., van de Bruck, C., Davis, A.-C., \& Rhodes, C. S. 2003, Ap\&SS, 283, 627

Burles, S., \& Tytler, D. 1998a, ApJ, 507, 732 1998b, ApJ, 499, 699

Castillo-Morales, A., \& Schindler, S. 2003, A\&A, 403, 433

Chamoun, N., Landau, S. J., \& Vucetich, H. 2001, Phys. Lett. B, 504, 1

Chamoun, N., \& Vucetich, H. 2002, Phys. Lett. B, 541, 291

Coc, A., Vangioni-Flam, E., Descouvemont, P., Adahchour, A., \& Angulo, C. 2004a, in AIP Conf. Proc. 704, Tours Symposium on Nuclear Physics V, ed. M. Arnould, et al. (Melville: AIP), 341 2004b, ApJ, 600, 544

Cottingham, W. 1963 , Ann. Phys., 25, 424

Cowie, L. L., \& Songaila, A. 1995, ApJ, 453, 596

Crighton, N. H. M., Webb, J. K., Ortiz-Gil, A., \& Fernández-Soto, A. 2004, MNRAS, 355, 1042

Cuoco, A., Iocco, F., Mangano, G., Miele, G., Pisanti, O., \& Serpico, P. D. 2004, Int. J. Mod. Phys. A, 19, 4431

Cyburt, R. H. 2004, Phys. Rev. D, 70(2), 023505

Cyburt, R. H., Fields, B. D., \& Olive, K. A. 2003, Phys. Lett. B, 567, 227

Damour, T., \& Dyson, F. 1996, Nucl. Phys. B, 480, 37

Damour, T., Piazza, F., \& Veneziano, G. 2002a, Phys. Rev. Lett., 89, 081601 2002b, Phys. Rev. D, 66, 046007

Damour, T., \& Polyakov, A. M. 1994, Nucl. Phys. B, 423, 532

Dmitriev, V. F., \& Flambaum, V. V. 2003, Phys. Rev. D, 67(6), 063513

Dmitriev, V. F., Flambaum, V. V., \& Webb, J. K. 2004, Phys. Rev. D, 69(6), 063506

Donahue, M., Gaskin, J. A., Patel, S. K., Joy, M., Clowe, D., \& Hughes, J. P. 2003, ApJ, 598, 190

Dyson, F. 1967, Phys. Rev. Lett., 19, 1291

Eidelman, S., et al. 2004, Phys. Lett. B, 592, 1

Epelbaum, E., Meißner, U., \& Glöckle, W. 2003, Nucl. Phys. A, 714, 535

Esmailzadeh, R., Starknam, G. D., \& Dimopoulos, S. 1991, ApJ, 378, 504

Ettori, S. 2003, MNRAS, 344, L13

Feng, J. L., Rajaraman, A., \& Takayama, F. 2003, Phys. Rev. D, 68(6), 063504

Flambaum, V. V. \& Shuryak, E. V. 2002, Phys. Rev. D, 65(10), 103503 2003, Phys. Rev. D, 67(8), 083507

Fowler, W. A., Caughlan, G. R., \& Zimmerman, B. A. 1967, ARA\&A, 5, 525 1975, ARA\&A, 13, 69

Freedman, W. L., et al. 2001, ApJ, 553, 47

Fujii, Y., Iwamoto, A., Fukahori, T., Ohnuki, T., Nakagawa, M., Hidaka, H., Oura, Y., \& Möller, P. 2000, Nucl. Phys. B, 573, 377 2002, preprint (hep-th/0205206)

Gleiser, M., \& Taylor, J. G. 1985, Phys. Rev. D, 31, 1904

Haugan, M. P., \& Will, C. M. 1976, Phys. Rev. Lett., 37, 1
Ichikawa, K., \& Kawasaki, M. 2002, Phys. Rev. D, 65, 123511 2004, Phys. Rev. D, 69(12), 123506

Ichikawa, K., Kawasaki, M., \& Takahashi, F. 2004, Phys. Lett. B, 597, 1

Ivanchik, A., Petitjean, P., Rodriguez, E., \& Varshalovich, D. 2002, Astron. Lett., 28 (423) 2003, Ap\&SS, 283, 583

Izotov, Y., \& Thuan, T. 1998, ApJ, 500, 188

Izotov, Y., Thuan, T., \& Lipovetsky, V. A. 1997, ApJS, 108, 1

Kaluza, T. 1921, Sitzungsber. Preussischen Akad. Wiss. Phys.-Math. Kl., 1, 966

Kawano, L. 1992, Let's Go: Early Universe. 2. Primordial Nucleosynthesis: The Computer Way (Fermilab Pub-92-004-A; Batavia: Fermilab)

Kirkman, D., Tytler, D., Suzuki, N., O’Meara, J. M., \& Lubin, D. 2003, ApJS, 149,1

Klein, O. 1926, Z. Phys., 37, 895

Landau, S. J., \& Vucetich, H. 2002, ApJ, 570, 463

Levshakov, S. A., Dessauges-Zavadsky, M., D’Odorico, S., \& Molaro, P. 2002, MNRAS, 333, 373

Maeda, K. 1988, Mod. Phys. Lett. A, 3, 243

Majerowicz, S., Neumann, D. M., \& Reiprich, T. H. 2002, A\&A, 394, 77

Marion, H., et al. 2003, Phys. Rev. Lett., 90, 150801

Martínez Fiorenzano, A. F., Vladilo, G., \& Bonifacio, P. 2003, Soc. Astron. Italiana Mem. Supp., 3, 252

Martins, C. J., Melchiorri, A., Trotta, R., Bean, R., Rocha, G., Avelino, P. P., \& Viana, P. T. 2002, Phys. Rev. D, 66, 023505

Mathews, G. J., Kajino, T., \& Shima, T. 2005, Phys. Rev. D, 71(2), 021302

Meléndez, J., \& Ramírez, I.: 2004, ApJ, 615, L33

Mukhanov, V. 2003, Int. J. Theor. Phys., 43, 669

Müller, C. M., Schäfer, G., \& Wetterich, C. 2004, Phys. Rev. D, 70(8), 083504

Murphy, M. T., Webb, J. K., \& Flambaum, V. V. 2003, MNRAS, 345, 609

Murphy, M. T., Webb, J. K., Flambaum, V. V., Dzuba, V. A., Churchill, C. W., Prochaska, J. X., Barrow, J. D., \& Wolfe, A. M. 2001a, MNRAS, 327, 1208

Murphy, M. T., Webb, J. K., Flambaum, V. V., Prochaska, J. X., \& Wolfe, A. M. 2001b, MNRAS, 327, 1237

Nollet, K. M., \& Lopez, R. E. 2002, Phys. Rev. D, 66, 063507

Olive, K. A., Pospelov, M., Qian, Y., Coc, A., Cassé, M., \& Vangioni-Flam, E. 2002, Phys. Rev. D, 66(4), 045022

Olive, K. A., \& Skillman, E. D. 2004, ApJ, 617, 29

Olive, K. A., \& Steigman, G. 1995, ApJS, 97, 49

O'Meara, J. M., Tytler, D., Kirkman, D., Suzuki, N., Prochaska, J. X., Lubin, D., \& Wolfe, A. M. 2001, ApJ, 552, 718

Overduin, J. M., Wesson, P. S. 1997, Phys. Rep., 283, 303

Palma, G. A., Brax, P., Davis, A. C., \& van de Bruck, C. 2003, Phys. Rev. D, 68, 123519

Peimbert, M., Peimbert, A., \& Ruiz, M. 2000, ApJ, 541, 688

Percival, W. J., et al. 2001, MNRAS, 327, 1297

Pessah, M. E., \& Torres, D. F. 2001, Phys. A, 297, 201

Pessah, M. E., Torres, D. F., \& Vucetich, H. 2001, Phys. A, 297, 164

Pettini, M., \& Bowen, D. V. 2001, ApJ, 560, 41

Pope, A. C., et al. 2004, ApJ, 607, 655

Prestage, J. D., Tjoelker, R. L., \& Maleki, L. 1995, Phys. Rev. Lett., 74, 3511

Quast, R., Reimers, D., \& Levshakov, S. A. 2004, A\&A, 415, L7

Richard, O., Michaud, G., \& Richer, J. 2005, ApJ, 619, 538

Riveros, C., \& Vucetich, H. 1986, Phys. Rev. D, 34, 321

Rocha, G., Trotta, R., Martins, C. J. A. P., Melchiorri, A., Avelino, P. P., \& Viana, P. T. P. 2003, NewA Rev., 47, 863

Romano, D., Tosi, M., Matteucci, F., \& Chiappini, C. 2003, MNRAS, 346, 295

Ryan, S., Beers, T., Olive, K., Fields, B. D., \& Norris, J. E. 2000, ApJ, 530, L57

Sisterna, P., \& Vucetich, H. 1990, Phys. Rev. D, 41, 1034

Smolliar, M. 1996, Science, 271, 1099

Sortais, Y., et al. 2000, Phys. Scr., T95, 50 
Spergel, D. N., et al. 2003, ApJS, 148, 175

Srianand, R., Chand, H., Petitjean, P., \& Aracil, B. 2004, Phys. Rev. Lett., 92, 121302

Stevenson, P. 1981, Ann. Phys., 132, 383

Torres, D. F., Vucetich, H., \& Plastino, A. 1997, Phys. Rev. Lett., 79, 1588

Varshalovich, D. A., Panchuk, V. E., \& Ivanchik, A. V. 1996, Astron. Lett., 22, 6

Wagoner, R. V. 1973, ApJ, 179, 343

Wagoner, R. V., Fowler, W. A., \& Hoyle, F. 1967, ApJ, 148, 3

Webb, J. K., Flambaum, V. V., Churchill, C. W., Drinkwater, M. J., and Barrow, J. D. 1999, Phys. Rev. Lett., 82, 884
Webb, J. K., Murphy, M. T., Flambaum, V. V., Dzuba, V. A., Barrow, J. D., Churchill, C. W., Prochaska, J. X., \& Wolfe, A. M. 2001, Phys. Rev. Lett., 87, 091301

Weinberg, S. 1983, Phys. Lett. B, 125, 265

Wu, Y., \& Wang, Z. 1986, Phys. Rev. Lett., 57, 1978

Youm, D. 2001a, Phys. Rev. D, 63, 125011

2001b, Phys. Rev. D, 64, 085011 